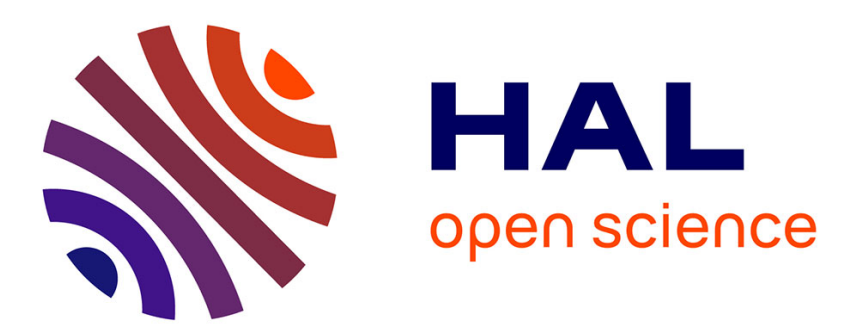

\title{
Hermite type Spline spaces over rectangular meshes with complex topological structures
}

\author{
Meng Wu, Bernard Mourrain, André Galligo, Boniface Nkonga
}

\section{To cite this version:}

Meng Wu, Bernard Mourrain, André Galligo, Boniface Nkonga. Hermite type Spline spaces over rectangular meshes with complex topological structures. Communications in Computational Physics, 2017, 21 (3), pp.835-866. 10.4208/cicp.OA-2016-0030 . hal-01196428v4

\section{HAL Id: hal-01196428 \\ https://hal.inria.fr/hal-01196428v4}

Submitted on 25 Apr 2017

HAL is a multi-disciplinary open access archive for the deposit and dissemination of scientific research documents, whether they are published or not. The documents may come from teaching and research institutions in France or abroad, or from public or private research centers.
L'archive ouverte pluridisciplinaire HAL, est destinée au dépôt et à la diffusion de documents scientifiques de niveau recherche, publiés ou non, émanant des établissements d'enseignement et de recherche français ou étrangers, des laboratoires publics ou privés. 


\title{
Hermite type Spline spaces over rectangular meshes with complex topological structures
}

\author{
Meng $\mathrm{Wu}^{3,1, *}$, Bernard Mourrain ${ }^{1}$, André Galligo ${ }^{2}$, and Boniface \\ Nkonga $^{2}$ \\ ${ }^{1}$ Galaad2, Inria, Sophia Antipolis, France. \\ 2 Lab. J. A. Dieudonné, University of Nice, Nice, France. \\ ${ }^{3}$ School of Mathematics, Hefei University of Technology, P. R. China.
}

\begin{abstract}
Motivated by the magneto hydrodynamic (MHD) simulation for Tokamaks with Isogeometric analysis, we present splines defined over a rectangular mesh with a complex topological structure, i.e., with extraordinary vertices. These splines are piecewise polynomial functions of bi-degree $(d, d)$ and $\mathcal{C}^{r}$ parameter continuity. And we compute their dimension and exhibit basis functions called Hermite bases for bicubic spline spaces. We investigate their potential applications for solving partial differential equations (PDEs) over a physical domain in the framework of Isogeometric analysis. For instance, we analyze the property of approximation of these spline spaces for the $L^{2}$-norm; we show that the optimal approximation order and numerical convergence rates are reached by setting a proper parameterization, although the fact that the basis functions are singular at extraordinary vertices.
\end{abstract}

AMS subject classifications: 65D05, 65U07, 65U30

Key words: Spline, meshes with complex topological structures, extraordinary vertices, Dimension and basis, Isogeometric analysis, MHD simulation.

\section{Introduction}

The finite element method (FEM) is a powerful tool that is often used to derive accurate and robust scheme for the approximation of the solution of PDEs. We are concerned with MHD equations applied to the edge plasma of fusion devices as Tokamaks. In this context of strongly magnetized plasma, the finite element formulation faces some difficulties such as the divergence-free constraint and the high anisotropy of transport processes.

Higher anisotropies suggest the use of meshes aligned with the principal directions of the transport processes [1]. Quadrilateral (2D) and hexahedral (3D) meshes, called

*Corresponding author. Email addresses: meng.wu@hfut.edu.cn, wumeng@mail.ustc.edu.cn (M. Wu), Bernard.Mourrain@inria.fr (B. Mourrain), Andre.Galligo@unice.fr (A. Galligo), boniface.nkonga@unice.fr (B. Nkonga) 
structured meshes, are the most convenient for alignment and lead to a reduction in the approximation error. In [2], isoparametric bicubic Hermite elements are used to solve the Grad-Shafranov equation, the equilibrium in the resistive MHD model, over a physical domain by aligning with concentric-circle-like principal directions of transport processes. They introduced a polar-coordinate-like transformation to construct a global coordinate system to achieve the desirable properties of the classical cubic Hermite element [3]. However, to align the principal directions of the transport processes in our target application for high-confinement Tokamaks, a structured mesh is involved, as shown on the left side of Figure 1. Different from a regular structured mesh, there is an extraordinary vertex (the $X$ point in Figure 1). In this paper, we present splines defined on a rectangular mesh with a complex topological structure, i.e. the mesh allows extraordinary vertices. To solve PDEs, the properties of approximation and numerical convergence rates of these splines are discussed.

NURBS, tensor product B-splines [4], hierarchical B-splines [5] [6] [7], LR-splines [8] and T-splines [9] are often used as shape functions to generate a parameterization. Their meshes are aligned with these directions by this parameterization. However, without an extraordinary vertex, their meshes are associated with simpler topological structures than that needed for the target application. In other words, the structured mesh on the left side of Figure 1 has to be decomposed when these splines are treated as shape functions. On the right side of Figure 1, we give an example of decomposition of the structured mesh into block structured meshes. However, the construction of high-quality block structured meshes is a challenging issue, even if isoparametric finite elements [10] or isogeometric analysis [11] can help to fit on physically curved principal directions of the transport processes.

To deal with extraordinary vertices, there are two classes of the definitions of splines. One is splines defined over a manifold, see e.g. [12-15]. That is based on the classical definition of a differential manifold. The crucial issue of constructing manifold splines in $[12,13]$ is obtaining an affine structure of a manifold. In other words, for a given physical domain, its parameterization is fixed if manifold splines are adopted. It doesn't consider the principal directions of transport processes for the target application. Another traditional way to solve extraordinary vertices problem is by a technique called geometric continuity. Several works focus on the construction of $G^{1}$ surfaces which are spline surfaces with tangent plane continuity, such as [16-24]. The ideas are using transition maps or reparameterizations. Especially, in [16], $C^{k}$-splines are defined over a mesh with a complex topological structure for the applications in geometric modelling. That definition generalizes the topological structure of meshes such that splines can be defined on meshes with more general topological structures.

For structured meshes aligned in the target application, they are not only determined by the geometry of a physical domain, but also the principal directions of the anisotropy transport processes. They are more easy to be aligned by splines defined on meshes with a parameterization, which is a linear combination of these splines. And based on this parameterization, we can solve PDEs over the physical domain directly in the framework 

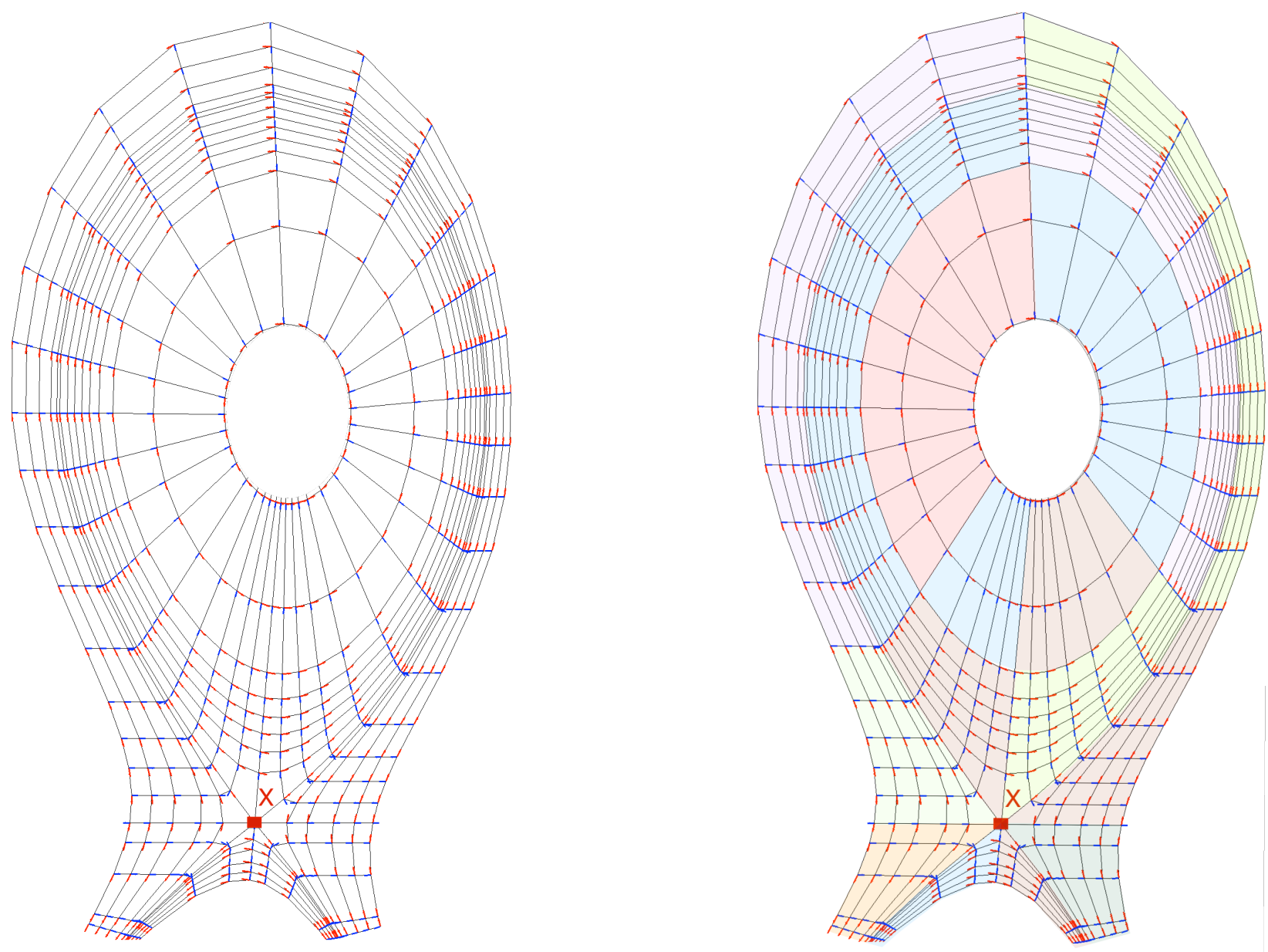

Figure 1: The structured mesh of the target application and its block structured mesh 
of Isogeometric analysis [11]. Thus, in this paper, we generalize " $C^{k}$-splines" in [16] to solving PDEs numerically. Concretely, the principle contributions of this paper are listed in the following:

1. The paper presents the definition of spline spaces over rectangular meshes with a complex topological structure. This definition extends " $C^{k}$-splines" on a mesh in [16] from geometry modelling to solving PDEs numerically. For instance, local refinement property is added to parametric meshes (see Remark 2.4) and the nesting property of spline spaces is discussed (see Notation 2.9). Moreover, to apply these splines to the target application, dimension formulas and basis functions called Hermite bases are provided for bicubic spline spaces with $C^{1}$ parameter continuity.

2. The approximation property of Hermite bases is presented. Although Hermite bases are singular at extraordinary vertices, the optimal approximation order is reached by setting a proper parameterization ( i.e., the parameterization satisfies the first point of Notation 4.1).

3. By isogeometric techniques, numerical convergence rates are tested for solving linear and nonlinear Grad-Shafranov equations. For the continuity of the numerical solution and its gradients, the optimal convergence rates measured by $L^{2}$-norm and $H^{1}$-norm are reached. By splines defined in this paper, a general physical domain with complex principal directions of transport processes can be described globally, not only for concentriccircle-like ones in [2].

The paper is structured as follows. From Section 2 to Section 3, we focus on defining this type of spline spaces, the dimension formulae and Hermite bases construction for a bicubic spline space with $\mathcal{C}^{1}$ parameter continuity. In Section 4 , the property of Hermite bases is discussed for solving PDEs over a parametric mesh with a complex topological structure in the framework of Isogeometric analysis. And we analyze the interpolation approximation error in Section 4.2. In Section 5, numerical convergence rates are measured by $L^{2}$-norm and $H^{1}$-norm for solving the Grad-Shafranov equations by isogeometric techniques. The last section proposes a conclusion of our work and directions for future works.

\section{Parametric meshes and spline spaces}

In this section, the fundamental definitions of this paper is presented. First, in Section 2.1, the concept of parametric meshes is given. Its definition includes an equivalence relation, which allows to address general topologies. Then the definition of spline spaces over a parametric mesh is introduced in Section 2.2.

\subsection{Parametric meshes}

In this section, we introduce the concept of a parametric mesh, which generalises the notion of a mesh in the parametric space, considered, e.g., in [16]. 
Informally, a parametric mesh is a collection of planar cells, that are glued together along edges with transition maps transforming the parameter values of one cell into parameter values on the other cell adjacent to the common edge. The precise definition of a parametric mesh is given is given in Definition 2.1. Before given the formal definition, we illustrate it in Example 2.1. The basic concepts about parametric meshes are presented in Definition 2.2 and they are illustrated in Example 2.2. In preparation for defining of spline spaces in Section 2.2 and discussing basis construction in Section 3, other concepts are introduced in Section 2.1.2, such as local frames, hanging vertices, basis vertices and composite edges of a parametric mesh. Section 2.1.3 introduces how to perform a local refinement of a parametric mesh. The definition of hierarchical parametric meshes is then presented, based on a local refinement process.

\subsubsection{Definition of Parametric Meshes}

Notation 2.1. The metric plane $\mathbb{R}^{2}$ equipped with coordinates $(s, t)$, will be called the $(s, t)$-plane. A cell is a rectangle of $\mathbb{R}^{2}$. We will use the letter $C$ with indices to denote cells. The boundary of a cell is decomposed into a finite set of segments (at least 4 but maybe more), called the edges of the cell. We will use the letter $e$ with indices to denote edges. The end points of these edges are called the vertices of the cell. We will use the letters $v$ or $w$ with indices to denote vertices. In other words, edges are segments between vertices.

We will consider an equivalence relation on the vertices (resp. edges) of a union of cells. Before providing a formal definition of our concept, let us illustrate it with a simple example to show a parametric mesh with a complex topological structure.

Example 2.1 (A parametric mesh and its topological structure). The left mesh of Figure 2 shows a parametric mesh $\mathscr{M}$. Its equivalent vertices have been marked with the same vertex labels and the edges between equivalent vertices are equivalent.

The topological structure of $\mathscr{M}$ is the same as the topology of a "usual" mesh, shown on the right side of Figure 2. From this example, a parametric mesh allows extraordinary vertices, whose valences are not 4 .
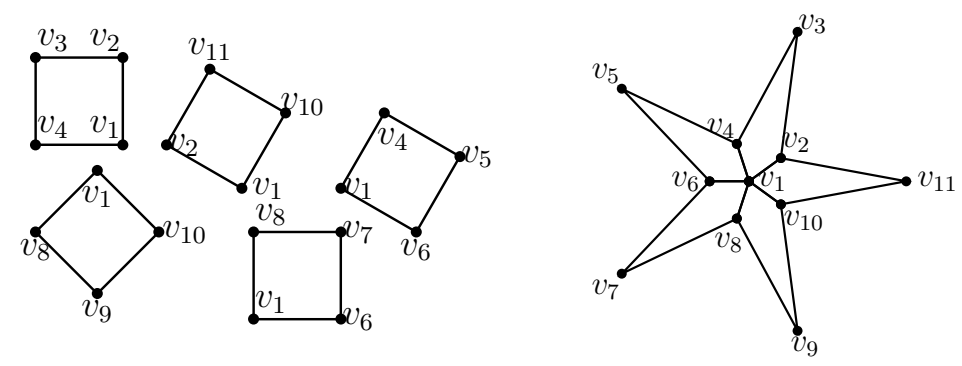

Figure 2: A parametric mesh $\mathscr{M}$ with 5 cells (left) and a "usual" mesh with the same topology of $\mathscr{M}$ 
Now, the formal definition of a parametric mesh is presented.

Definition 2.1 (A Parametric Mesh). A parametric mesh $\mathscr{M}$ is given by a collection of cells denoted by $C_{1}, C_{2}, \cdots, C_{N}$, a subset $\mathscr{P}_{F}$ of $\mathrm{I}=\{\{i, j\}: i, j \in\{1,2, \cdots, N\}$ and $i \neq j\}$, a collection of transition maps indexed by $\mathscr{P}_{F}$, and an equivalence relation " $\sim$ " on the edges and vertices of the cells, satisfying the following properties.

1. For each pair $(i, j) \in \mathscr{P}_{F}$, there exists a pair of transition maps:

- $\phi_{i, j}: C_{i} \rightarrow \mathbb{R}^{2}$,

- $\phi_{j, i}: C_{j} \rightarrow \mathbb{R}^{2}$

and a pair of edges $e_{i, j}$ of $C_{i}$ and $e_{j, i}$ of $C_{j}$ such that

- $\phi_{i, j}\left(C_{i}\right) \cap C_{j}=e_{j, i}$,

- $\phi_{j, i}\left(C_{j}\right) \cap C_{i}=e_{i, j}$,

- $\left.\phi_{i, j}\right|_{e_{i, j}}: e_{i, j} \longrightarrow e_{j, i},\left.\phi_{j, i}\right|_{e_{j, i}}: e_{j, i} \longrightarrow e_{i, j}$ are diffeomorphisms and $\left(\left.\phi_{i, j}\right|_{e_{i, j}}\right)^{-1}=\left.\phi_{j, i}\right|_{e_{j, i}}$.

Then, $e_{i, j} \sim e_{j, i}$.

Moreover, for any two vertices $v$ of $e_{i, j}$ and $w$ of $e_{j, i}$, if $\phi_{i, j}(v)=w$ then $v \sim w$.

2. For any edge $e$ of $C_{i}(i=1,2, \cdots, n)$, the number of edges that are equivalent to $e$ by " $\sim$ " is no more than 2 .

Remark 2.2 (A Parametric Mesh). The main difference with manifold spline constructions $[12,13]$ is that we do not require an atlas of an explicit manifold but only transition maps between the adjacent cells of a parametric mesh. The existence of a manifold is not required.

Definition 2.2 (Interior vs Boundary, degree). The equivalence class of edges and vertices of $C_{i}$ are called $\mathscr{M}^{\prime}$ s edges and vertices. If an edge equivalence class has two elements, then it is called an interior edge of $\mathscr{M}$; otherwise it is called a boundary edge. If a vertex is on a boundary edge, then it is called a boundary vertex; otherwise, it is called an interior vertex. The degree $\operatorname{deg}(v)$ of an equivalence class of vertices $v$ is the number of distinct equivalence classes of edges $e$ containing $v$.

Example 2.2 (Interior vs Boundary, degree). In Example 2.1, note that, e.g., $v_{6} v_{7}$ is a boundary edge, while $v_{1} v_{2}$ and $v_{1} v_{6}$ are interior edges (because they are shared by two cells). We have $\operatorname{deg}\left(v_{1}\right)=5, \operatorname{deg}\left(v_{2}\right)=3$ and $\operatorname{deg}\left(v_{3}\right)=2$.

Definition 2.3 (Equivalent parametric meshes). Now consider two adjacent cells $C_{1}$ and $C_{2}$ of $\mathscr{M}$, (i.e., that share a common edge). 

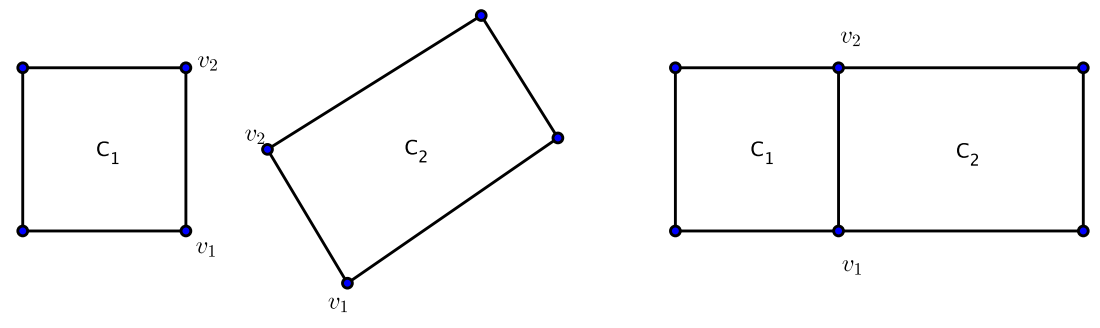

Figure 3: Transition maps

They can be moved together by the transition map, which is a rigid transformation. This is illustrated in Figure 3. By this way, $\mathscr{M}$ becomes another parameter mesh $\mathscr{M}^{\prime}$ with identity transition maps between two adjacent cells, where $\mathscr{M}^{\prime}$ is called an equivalent parametric mesh of $\mathscr{M}$.

Example 2.3 (Equivalent parametric meshes). A planar T-mesh, as defined in [25], can be represented by a parametric mesh such as the right side of Figure 4, where all of the transition maps are identity maps.

In the left side of Figure 4 there is another parametric mesh with all of the transition maps, which are rigid transformations. The T-mesh in the right side of Figure 4 is an equivalent parametric mesh of the parametric mesh in the left side of Figure 4.
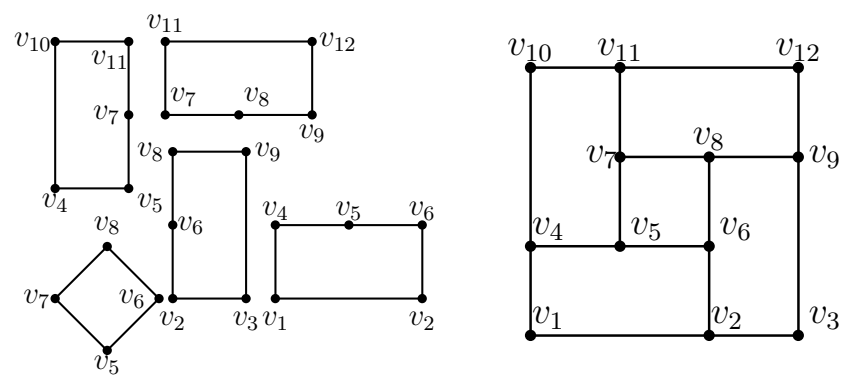

Figure 4: A parametric mesh with rigid transformations and its equivalent parametric mesh with identity transition maps

\section{Remark 2.3 (Restrictions).}

- A parametric mesh can also describe a non-orientable surface, such as the Moebius strip. However, in this article, we will restrict ourselves to the case where $\mathscr{M}$ is orientable. Furthermore, we suppose that any edge (resp. vertex) of a cell is not equivalent to another edge (resp. vertex) of the same cell. 
- We also assume that an edge is shared by no more than two faces.

\subsubsection{Local frames, hanging vertices, basis vertices and composite edges of a paramet- ric mesh}

In this section, we introduce local frames (Definition 2.4), hanging vertices, basis vertices and composite edges (Definition 2.5) of a parametric mesh. These concepts are used to describe spline spaces in Section 2.2, dimension formulae and basis construction in Section 3.

Definition 2.4 (Local frame). Let $\mathscr{M}$ be a parametric mesh and denote its cells as $C_{1}, C_{2}, \cdots, C_{N}$. For each cell $C_{i}, i=1 \ldots N$, we define a local frame by two unit vectors $\mathscr{F}_{i}:=\left(\mathbf{s}_{i}, \mathbf{t}_{i}\right)$ that parallel each of the two directions of the edges of $C_{i}$ (and that agrees with the metric of the $(s, t)$-plane). This is illustrated in Figure 5.

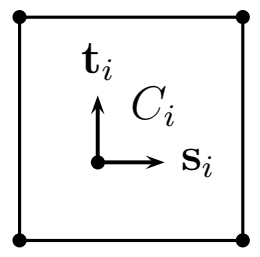

Figure 5: A local frame of $C_{i}$

Definition 2.5 (Hanging vertices, Basis vertices and Composite edges).

- An interior vertex $v$ of $\mathscr{M}$ is called a hanging vertex if there is a cell $C$ such that $v$ is on an edge of $C$ and it is not a corner point of $C$.

- A vertex of $\mathscr{M}$ that is not a hanging vertex is called a basis vertex.

- A composite edge of $\mathscr{M}$ is the longest possible "line segment" that consists of several interior edges, and each non-end vertex of this "line segment" is a hanging vertex of $\mathscr{M}$, where if two edges locate on the same line or not, this point is determined by their positions in $\mathscr{M}^{\prime}$ s equivalent parametric mesh.

Example 2.4 (Hanging vertices, Basis vertices and Composite edges). In the left side of Figure 4 , the vertices $v_{5}, v_{7}, v_{6}$ and $v_{8}$ are hanging vertices. $v_{9}$ is not a hanging vertex. Because it is a boundary vertex and it is a corner point of a cell. $v_{5} v_{7}$ is an interior edge, and $v_{5} v_{11}$ is a composite edge that includes two edges $v_{5} v_{7}$ and $v_{7} v_{11}$. Because $v_{5} v_{11}$ lies on the same line of its equivalent parametric mesh (T-mesh), shown in the right side of Figure 4, it satisfies the definition of "a composite edge".

Remark 2.4. If $\mathscr{M}$ has no hanging vertices and all of its cells are unit squares, it is called a spline domain, as defined in [16]. 


\subsubsection{Local Refinement and Hierarchical parametric meshes}

In adaptive solving PDEs procedure, refinement is an important operation. Traditionally, one distinguishes between two types of refinement: h-refinement and p-refinement. The first one, also called h-adaptivity, amounts to splitting elements in space while keeping their polynomial degree fixed, whereas p-adaptivity amounts to increasing the polynomial degrees. Hereafter, a simple scheme of the local h-refinement of $\mathscr{M}$ is presented.

Definition 2.6 (Local Refinement Rule). Let $\mathscr{M}$ be a parametric mesh. A refined parametric mesh $\mathscr{M}^{\prime}$ is obtained by splitting some of the cells of $\mathscr{M}$ along lines parallel to one of the edges of these cells. The transition maps of $\mathscr{M}^{\prime}$ are defined as follows.

- Given two cells $C_{i}$ and $C_{j}$ of $\mathscr{M}$ with an adjacent edge $e_{i, j} \sim e_{j, i}$ which is split, for any cells $C_{i^{\prime}}^{\prime} \subset C_{i}, C_{j^{\prime}}^{\prime} \subset C_{j}$ of $\mathscr{M}^{\prime}$ with a common sub-edge of $e_{i, j} \sim e_{j, i}$, the transition map between $C_{i^{\prime}}^{\prime}$ and $C_{j^{\prime}}^{\prime}$ is the restriction respectively to $C_{i^{\prime}}^{\prime}$ and $C_{j^{\prime}}^{\prime}$ of the transition $\operatorname{maps} \phi_{i, j}: C_{i} \longrightarrow \mathbb{R}^{2}$ and $\phi_{j, i}: C_{j} \longrightarrow \mathbb{R}^{2}$.

- For a cell $C$ of $\mathscr{M}$ split into sub-cells $C_{1}^{\prime}, C_{2}^{\prime}$ of $\mathscr{M}^{\prime}$ along an edge $e^{\prime}$, the transition map across $e^{\prime}$ is the identity.

This refinement construction can be iterated. Notice that the refinements create additional hanging vertices.

Definition 2.7 (Hierarchical parametric mesh). A hierarchical parametric mesh $\mathscr{M}$ is a mesh obtained by the iterated refinement of an initial parametric mesh, where we will also assume that the initial mesh has no hanging vertex.

If the initial mesh is just a cell, $\mathscr{M}$ is a hierarchical T-subdivision, as described in [31].

\subsection{Spline spaces over a parametric mesh}

In this section, based on the definition of a parametric mesh in Section 2.1, we define the spline space over a parametric mesh in Section 2.2.1. Formally, this definition depends on the choice of local frames. In Section 2.2.2, the local frame independency is checked. After that, in Section 2.2.3, the nesting property of spline spaces is presented by the local refinement of parametric meshes. This property is usually required in adaptive solving PDEs procedure.

\subsubsection{Definition of spline spaces over a parametric mesh}

Definition 2.8 (Spline over $\mathscr{M}$ ). A spline $f$ of bi-degree $(d, d)$ and $\mathcal{C}^{r}$ regularity over $\mathscr{M}$ with the local frame set $\mathscr{F}=\left\{\mathscr{F}_{i}\right\}$ is given by a collection of polynomials $f_{i}$ satisfying 1. $f_{i}\left(s_{i}, t_{i}\right):=\left.f\right|_{C_{i}} \in \mathbb{R}_{d, d}\left[s_{i}, t_{i}\right], i=1, \ldots, N$, where $\left(s_{i}, t_{i}\right)$ is the coordinates associated with the given local frame $\mathscr{F}_{i}$ of $C_{i}$;

2. If $v_{i, j} \sim v_{j, i}$ is a (class) of vertex common to cells $C_{i}$ and $C_{j}$ of $\mathscr{M}$, then

$$
f_{i}\left(v_{i, j}\right)=f_{j}\left(v_{j, i}\right) \text {. }
$$


3. If $e_{i, j} \sim e_{j, i}$ is a (class) of edge common to cells $C_{i}$ and $C_{j}$ of $\mathscr{M}$, then $f_{i}\left(s_{i}, t_{i}\right)$ and $f_{j}\left(s_{j}, t_{j}\right)$ are " $\mathrm{C}^{r}$ across the edge".

More precisely, let $\phi_{i, j}, \phi_{j, i}$ be the transition maps between $C_{i}$ and $C_{j}$, such that $\phi_{i, j}\left(e_{i, j}\right)=$ $e_{j, i} ;$ denote as $\mathbf{n}_{i, j}$ and $\mathbf{n}_{j, i}$ unit vectors of the metric $(s, t)$-plane, perpendicular, respectively, to $e_{i, j}$ and $e_{j, i}$. Then, we must have

$$
\begin{aligned}
& \left.D_{\mathbf{n}_{i, j}}^{k}\left(f_{j} \circ \phi_{j, i}^{-1}\right)\right|_{e_{i, j}}=\left.D_{\mathbf{n}_{i, j}}^{k} f_{i}\right|_{e_{i, j}} \\
& \left.D_{\mathbf{n}_{j, i}}^{k}\left(f_{i} \circ \phi_{i, j}^{-1}\right)\right|_{e_{j, i}}=\left.D_{\mathbf{n}_{j, i}}^{k} f_{j}\right|_{e_{j, i}}
\end{aligned}
$$

for $k=0,1,2, \cdots, r$.

Remark 2.5 (Spline over $\mathscr{M}$ ). By Equations (2.1) (2.2), splines are $\mathcal{C}^{r}$ in the parameters $\left(s_{i}, t_{i}\right)$ across the common edge of two adjacent cells associated with $\phi_{i, j}, \phi_{j, i}$. For rigid transformation maps that are $\mathcal{C}^{k}$-diffeomorphims, conditions (2.1) and (2.2) are equivalent. In this paper, from Section 3, we focus on the bi-cubic spline spaces with rigid transformation maps. For spline spaces with more general transformation maps, we refer to [33].

Remark 2.6 (Spline over $\mathscr{M}$ vs $\mathcal{C}^{r}$-splines in [16]). If the transition maps $\phi_{i, j}, \phi_{j, i}$ are rigid transformations and all the cells $C_{i}$ are unit squares, then the splines defined here are $\mathcal{C}^{r}$-splines in [16].

Notation 2.7 (Basic properties of Spline over $\mathscr{M}$ ). We denote by $\mathbf{S}(d, r ; \mathscr{M}$ ) the set of splines defined in Definition 2.8. We call it a spline space over $\mathscr{M}$.

We have:

- By linearity of directional derivatives, $\mathbf{S}(d, r ; \mathscr{M})$ is a vector space;

- It is finite dimensional;

- $1 \in \mathbf{S}(d, r ; \mathscr{M})$.

\subsubsection{Spline space and local frames}

Let us discuss the dependency of the spline space $\mathscr{M}$ on the choice of local frames $\mathscr{F}_{i}$ for the cells $C_{i}$.

Considering another local frame $\mathscr{F}_{i}^{\prime}$, there exists a set of orthogonal transformations $\mathbf{O}=\left\{\mathbf{O}_{i}\right\}$ such that

$$
\mathscr{F}_{i}=\mathbf{O}_{i} \mathscr{F}_{i}^{\prime}, i=1 \ldots N \text {. }
$$

By the action of $\mathbf{O}_{i}$, any polynomial in the coordinates of $\mathscr{F}_{i}$ becomes a polynomial in the coordinates of $\mathscr{F}_{i}^{\prime}$ of the same degree. Moreover, the other two items in Definition 2.8, evaluation at a vertex and regularity, are conserved when expressed in the other frame. This proves the following theorem. 


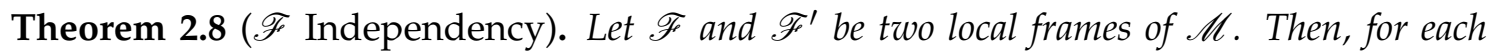
cell $C_{i}, i=1 \ldots N$, of $\mathscr{M}$, there exists an orthogonal transformation $\mathbf{O}_{i}$ sending $\mathscr{F}_{i}^{\prime}$ to $\mathscr{F}_{i}, i$. e., $\mathscr{F}_{i}=\mathbf{O}_{i} \mathscr{F}_{i}^{\prime}$. If $f \in \mathbf{S}(d, r ;\langle\mathscr{M}, \mathscr{F}\rangle)$, so that

$$
f \circ \mathbf{O} \in \mathbf{S}\left(d, r ;\left\langle\mathscr{M}, \mathscr{F}^{\prime}\right\rangle\right),
$$

where $\left.\mathbf{O}\right|_{\mathscr{F}}=\mathbf{O}_{i},\left.f \circ \mathbf{O}\right|_{C_{i}}=f\left(\mathbf{O}_{i}\left(s_{i}^{\prime}, t_{i}^{\prime}\right)\right)$, where $\mathbf{S}(d, r ;\langle\mathscr{M}, \mathscr{F}\rangle)$ is a spline space over $\mathscr{M}$ with the local frames $\mathscr{F}$. Its bi-degree is $(d, d)$ and, it has $\mathcal{C}^{r}$ regularity.

Thus, up to a set of orthogonal transformations, splines over $\mathscr{M}$ are independent of the choice of $\mathscr{F}$.

\subsubsection{Local refinement and spline spaces}

Let $\mathscr{M}^{\prime}$ be a parametric mesh obtained by refining some cells of $\mathscr{M}$. Then, a spline function in $\mathbf{S}(d, r ; \mathscr{M})$ is a spline function defined over the refined mesh $\mathscr{M}^{\prime}$, by the local refinement rule in Definition 2.6.Thus, we have:

Notation 2.9 (The Nesting Property of spline spaces).

$$
1 \in \mathbf{S}(d, r ; \mathscr{M}) \subseteq \mathbf{S}\left(d, r ; \mathscr{M}^{\prime}\right) .
$$

\section{Bicubic spline spaces over a parametric mesh}

In this section, bicubic spline spaces over a parametric mesh will be considered. We also consider transition maps that are rigid transformations of the plane. The main results in this section are the dimension formula and an explicit basis construction, where Hermite data play a crucial role.

Let $\mathscr{M}$ be a hierarchical mesh. The bicubic spline spaces over $\mathscr{M}$ is $\mathbf{S}(3,1 ; \mathscr{M})$. Here, we organize this section as follows. In Section 3.1, the Hermite data of a bicubic spline over a parametric mesh are discussed. Based on the analysis of Section 3.1, in Section 3.2, the dimension formula of $\mathbf{S}(3,1 ; \mathscr{M})$ and a set of bases are presented.

\subsection{Hermite data}

In this section, a linear map $\mathscr{H}$ is introduced. By $\mathscr{H}$, a spline in $\mathbf{S}(3,1 ; \mathscr{M})$ is described by its Hermite data at each vertex of $\mathscr{M}$. Then, the constraints between its Hermite data are given, such that the constraints across a common edge (Section 3.1.1) become the constraints of the Hermite data at a basis vertex and hanging vertex (Section 3.1.2). By these constraints, $\mathscr{H}$ is reduced to a new injective map $\widetilde{\mathscr{H}}$.

We first illustrate the Hermite data construction on a single square. Let $Q$ be the square in the parametric $(s, t)$-plane with vertices $v_{1}:=[0,0], v_{2}:=[0,1], v_{3}:=[1,0]$, and $v_{4}:=[1,1]$. The vector space $E$ of polynomials of bidegree $(3,3)$ on $Q$ has dimension 16 and a basis of $E$ is formed by the two by two products of Bernstein polynomials $B_{i}^{3}(s)$ and $B_{j}^{3}(t)$ for $0 \leq i, j \leq 3$. 
We set

$$
H_{(s, t)}^{v_{\ell}}(f)=\left(f\left(v_{\ell}\right), \frac{\partial f}{\partial s}\left(v_{\ell}\right), \frac{\partial f}{\partial t}\left(v_{\ell}\right), \frac{\partial^{2} f}{\partial s \partial t}\left(v_{\ell}\right)\right)
$$

for $1 \leq \ell \leq 4$.

The 4 Hermite data $H_{\left(s_{i}, t_{i}\right)}^{v_{\ell}}(f)$ at each of the 4 vertices form 16 real numbers naturally associated with an element $f$ of $E$.

Lemma 3.1. The linear map $E \longrightarrow \mathbb{R}^{16}$ defined by the Hermite data at the 4 vertices of $Q$ is an isomorphism.

Proof. It suffices to check the non vanishing of the determinant of the corresponding matrix on the basis $B_{i}^{3}(s) B_{j}^{3}(t), 0 \leq i, j \leq 3$.

Notice that $H^{v_{\ell}}(f)$ depends only on the four Bernstein coefficients that are near $v_{\ell}$ as in the B-net method described in [25]. By this correspondence, the dimension formula and basis construction of $\mathbf{S}(3,1 ; \mathscr{M})$ are discussed by generalizing the B-net method in [25].

Let $\mathscr{M}^{\prime}$ s cells be $C_{1}, \ldots, C_{N}$. We extend the definition of the Hermite data to the spline space $\mathbf{S}(3,1 ; \mathscr{M})$ and via the following map:

$$
\begin{aligned}
\mathscr{H}: \mathbf{S}(3,1 ; \mathscr{M}) & \rightarrow \mathbb{R}^{4 N} \\
f & \mapsto\left(H_{\left(s_{i}, t_{i}\right)}^{v^{i}}\left(f_{i}\right)\right)_{i=1, \ldots, N, \ell=1, \ldots, 4}
\end{aligned}
$$

where $v_{\ell}^{i}, \ell=1, \ldots, 4$ are the 4 vertices of cell $C_{i}$ and $H_{\left(s_{i}, t_{i}\right)}^{v_{i}^{i}}\left(f_{i}\right)$ are the Hermite data of $f$ at $v_{\ell}^{i}$.

Lemma 3.2. The Hermite data map $\mathscr{H}$ defined in (3.1) is an injective linear map from $\mathbf{S}(3,1 ; \mathscr{M})$ to $\mathbb{R}^{4 N}$.

Proof. By construction, $\mathscr{H}$ is linear. To prove that it is injective, we note that if all Hermite data of a polynomial $f_{i}$ at the vertices of a cell $C_{i}$ are zero, then by Lemma 3.1, $f_{i} \equiv 0$. Thus, if $f \in \mathbf{S}(3,1 ; \mathscr{M})$ is such that $\mathscr{H}(f)=0$ then $f=0$. $\mathscr{M}$.

We now analyze different constraints applicable on the Hermite data at a vertex of

\subsubsection{Hermite data across a common edge}

In this section, we describe a $\mathcal{C}^{1}$ regularity condition across an edge in terms of Hermite data.

Lemma 3.3. Let $C_{1}, C_{2}$ be two cells with the common edge $e_{1,2}=v_{1} v_{2}$. Assume that they share the same local frame $(\mathbf{s}, \mathbf{t})$ and the same parameters $(s, t)$. Then $f_{1}(s, t)$ and $f_{2}(s, t)$ are $\mathcal{C}^{1}$ across $e_{1,2}$ if and only if their Hermite data at $v_{1}$ and $v_{2}$ coincide:

$$
H_{(s, t)}^{v_{\ell}}\left(f_{1}\right)=H_{(s, t)}^{v_{\ell}}\left(f_{2}\right), l=1,2 .
$$


Proof. Suppose that the edge $e_{1,2}$ is along the $t$-direction (the other case can be treated symmetrically). Because $f_{1}(s, t)$ and $f_{2}(s, t)$ are polynomials of bi-degree $(3,3)$, if their Hermite data coincide at $v_{1}$ and $v_{2}$, then we have $f_{1}(s, t)=f_{2}(s, t)$ and $\partial_{s} f_{1}(s, t)=\partial_{s} f_{2}(s, t)$ along the edge $v_{1} v_{2}=e_{1,2}$. In other words, the function defined by $\left(f_{1}, f_{2}\right)$ is $\mathcal{C}^{1}$ across the edge $e_{1,2}$

Conversely, if $\left(f_{1}, f_{2}\right)$ is $\mathcal{C}^{1}$ across the edge $e_{1,2}$, then their Hermite data at $v_{1}$ and $v_{2}$ must coincide.

Assume now that $C_{1}$ and $C_{2}$ have different frames, denoted by $\mathscr{F}_{1}=\left(\mathbf{s}_{1}, \mathbf{t}_{1}\right)^{T}$ and $\mathscr{F}_{2}=$ $\left(\mathbf{s}_{2}, \mathbf{t}_{2}\right)^{T}$. There must exist an orthogonal transformation $\mathbf{O}_{2,1}$, more precisely a rotation of angle $k \pi / 2(k \in \mathbb{Z})$, such that

$$
\mathscr{F}_{2}=\mathbf{O}_{2,1} \mathscr{F}_{1}, \mathbf{O}_{2,1}=\left(\begin{array}{cc}
O_{11} & O_{12} \\
O_{21} & O_{22}
\end{array}\right)
$$

The following lemma describes the relations between $H_{\left(s_{1}, t_{1}\right)}^{v_{\ell}}\left(f_{1}\right)$ and $H_{\left(s_{2}, t_{2}\right)}^{v_{\ell}}\left(f_{2}\right)$.

Lemma 3.4. $f_{1}\left(s_{1}, t_{1}\right)$ and $f_{2}\left(s_{2}, t_{2}\right)$ have $a \mathcal{C}^{1}$ fit if and only if

$$
H_{\left(s_{2}, t_{2}\right)}^{v_{\ell}}\left(f_{2}\right)^{T}=A H_{\left(s_{1}, t_{1}\right)}^{v_{\ell}}\left(f_{1}\right)^{T},
$$

where

$$
A=\left(\begin{array}{cccc}
1 & 0 & 0 & 0 \\
0 & O_{11} & O_{12} & 0 \\
0 & O_{21} & O_{22} & 0 \\
0 & 0 & 0 & O_{11} O_{22}+O_{12} O_{21}
\end{array}\right)
$$

where $O_{i, j}(i, j=1,2)$ are defined in (3.2).

Proof. By Lemma 3.3, after applying the transition map, we have $H_{\left(s_{1}, t_{1}\right)}^{v_{\ell}}\left(f_{1}\right)^{T}=H_{\left(s_{2}, t_{2}\right)}^{v_{\ell}}\left(f_{2}\right)^{T}$. Thus, we just considered the Hermite data at $v_{\ell}$ of $f_{2}$ using different frames $\mathscr{F}_{1}, \mathscr{F}_{2}$. Based on the fact that $\mathbf{O}_{2,1}$ in (3.2) is an orthogonal rotation of angle $k \pi / 2$, we explicitly computed matrix $A$ and obtained formula (3.4).

Remark 3.1. Because $\mathbf{O}_{2,1}$ in (3.2) is a rotation of angle $k \pi / 2$, we have $O_{11} O_{22}+O_{12} O_{21}=$ $(-1)^{k}$; consequently $\operatorname{rank}(A)=4$.

This shows that the Hermite data at $v_{\ell}$ on $C_{2}$ is uniquely determined by the Hermite data at $v_{\ell}$ on $C_{1}$, via the linear invertible transformation $A$. In this case we will say that the Hermite data at $v_{\ell}$ on $C_{1}$ and $C_{2}$ are compatible. 


\subsubsection{Hermite data at a basis vertex of degree $n$ and a hanging vertex}

Let $v$ be a basis vertex of $\mathscr{M}$ and $n$ be the degree of $v$. This means that there are $n$ cells $C_{1}, \ldots, C_{n}$ with $v$ as one of their corner vertices. These cells form $v$ 's 1-neighborhood. Let us consider the behavior of $f \in \mathbf{S}(3,1 ; \mathscr{M})$ at $v$.

We can assume that we have already sorted the 1-neighborhood of $v$ such that two successive cells share a common edge of $\mathscr{M}$ in a clockwise direction.

Given a local frame $\mathscr{F}_{1}=\left(\mathbf{s}_{1}, \mathbf{t}_{1}\right)^{T}$ of $C_{1}$, the local frame $\mathscr{F}_{k}$ of $C_{k}$ is defined as

$$
\mathscr{F}_{k}=\mathbf{O}_{k, 1} \mathscr{F}_{1},
$$

where

$$
\mathbf{O}_{k, 1}=\left(\begin{array}{cc}
\cos (\pi(k-1) / 2) & -\sin (\pi(k-1) / 2) \\
\sin (\pi(k-1) / 2) & \cos (\pi(k-1) / 2)
\end{array}\right)
$$

According to $\mathcal{C}^{1}$ continuity, the Hermite data at $v$ of $C_{k}$ is obtained by Eq. (3.2) and Eq. (3.3), i.e.,

$$
H_{\left(s_{k}, t_{k}\right)}^{v}\left(f_{k}\right)^{T}=A_{k} H_{\left(s_{1}, t_{1}\right)}^{v}\left(f_{1}\right)^{T},
$$

where $f_{i}=\left.f\right|_{C_{i}}, A_{k}$ is determined by $\mathbf{O}_{k, 1}$, similarly to Eq. (3.3). Because $v$ is a basis vertex, it can be a boundary vertex as well as an interior (not hanging) vertex. If $v$ is a boundary vertex of $\mathscr{M}$, the Hermite data of $H_{\left(s_{k}, t_{k}\right)}^{v}(f)(k>1)$ are well determined when $H_{\left(s_{1}, t_{1}\right)}^{v}(f)$ is given because $\operatorname{rank}\left(A_{k}\right)=4$.

Proposition 3.1. With the previous notations, if $v$ is an interior basis vertex and $n$ is its degree, we have

1. If $n \bmod 4=1$, then,

$$
\frac{\partial f(v)}{\partial s_{1}}=0, \frac{\partial f(v)}{\partial t_{1}}=0, \frac{\partial^{2} f(v)}{\partial s_{1} \partial t_{1}}=0 ;
$$

2. If $n \bmod 4=2$, then

$$
\frac{\partial f(v)}{\partial s_{1}}=0, \frac{\partial f(v)}{\partial t_{1}}=0
$$

3. If $n \bmod 4=3$, then

$$
\frac{\partial f(v)}{\partial s_{1}}=0, \frac{\partial f(v)}{\partial t_{1}}=0, \frac{\partial^{2} f(v)}{\partial s_{1} \partial t_{1}}=0
$$

Proof. If $v$ is an interior vertex of $\mathscr{M}$ and its degree is $n$. Consider $C_{1}$ and $C_{n}$ stick together clockwise, by (3.6),

$$
H_{\left(s_{n}, t_{n}\right)}^{v}\left(f_{n}\right)^{T}=A_{n} H_{\left(s_{1}, t_{1}\right)}^{v}\left(f_{1}\right)^{T},
$$




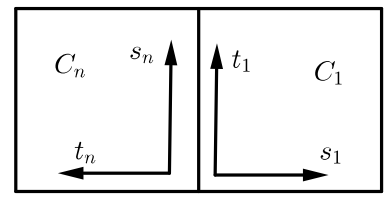

Figure 6: $C_{1}$ and $C_{n}$ stick anticlockwise

where $H_{\left(s_{i}, t_{i}\right)}^{v}\left(f_{j}\right)$ is the Hermite data at $v$ on $C_{j}$ with respect to the local frame $\left(\mathbf{s}_{i}, \mathbf{t}_{i}\right)$, $f_{j}=\left.f\right|_{C_{j}}, A_{n}$ is defined in (3.6).

Considering $C_{1}$ and $C_{n}$ stick together anticlockwise, the common edge is along $\mathbf{t}_{1}$ of $C_{1}$ and $\mathbf{s}_{n}$ of $C_{n}$ (shown in Figure 6). Then

$$
\left(\begin{array}{l}
\mathbf{s}_{1} \\
\mathbf{t}_{1}
\end{array}\right)=\left(\begin{array}{cc}
0 & -1 \\
1 & 0
\end{array}\right)\left(\begin{array}{l}
\mathbf{s}_{n} \\
\mathbf{t}_{n}
\end{array}\right)
$$

Thus, $H_{\left(s_{1}, t_{1}\right)}^{v}\left(f_{n}\right)^{T}=B H_{\left(s_{n}, t_{n}\right)}^{v}\left(f_{n}\right)^{T}$ and by (3.7),

$$
H_{\left(s_{1}, t_{1}\right)}^{v}\left(f_{n}\right)^{T}=B A_{n} H_{\left(s_{1}, t_{1}\right)}^{v}\left(f_{1}\right)^{T},
$$

where $B=\left(\begin{array}{cccc}1 & 0 & 0 & 0 \\ 0 & 0 & -1 & 0 \\ 0 & 1 & 0 & 0 \\ 0 & 0 & 0 & -1\end{array}\right)$.

$f_{1}$ and $f_{n}$ are $\mathcal{C}^{1}$ across the common edge with $v$ as their common vertex. By Lemma 3.3, $H_{\left(s_{1}, t_{1}\right)}^{v}\left(f_{n}\right)=H_{\left(s_{1}, t_{1}\right)}^{v}\left(f_{1}\right)$, i.e.,

$$
B A_{n} H_{\left(s_{1}, t_{1}\right)}^{v}\left(f_{1}\right)^{T}=H_{\left(s_{1}, t_{1}\right)}^{v}\left(f_{1}\right)^{T} .
$$

Thus $\left(I-B A_{n}\right) H_{\left(s_{1}, t_{1}\right)}^{v}\left(f_{1}\right)^{T}=0$.

When $n \bmod 4=1, I-B A_{n}=\left(\begin{array}{cccc}0 & 0 & 0 & 0 \\ 0 & 1 & 1 & 0 \\ 0 & -1 & 1 & 0 \\ 0 & 0 & 0 & 2\end{array}\right)$;

when $n \bmod 4=2, I-B A_{n}=\left(\begin{array}{cccc}0 & 0 & 0 & 0 \\ 0 & 2 & 0 & 0 \\ 0 & 0 & 2 & 0 \\ 0 & 0 & 0 & 0\end{array}\right)$;

when $n \bmod 4=3, I-B A_{n}=\left(\begin{array}{cccc}0 & 0 & 0 & 0 \\ 0 & 1 & -1 & 0 \\ 0 & 1 & 1 & 0 \\ 0 & 0 & 0 & 2\end{array}\right)$. 
The result of this proposition is obtained.

We recall that, by Definition 2.5, a hanging vertex $v$ is a non end vertex of a composite edge, which belongs to the interior of a segment joining two corner points of a cell. For hanging vertices, similar to Theorem 4.2 in [25], we get

Lemma 3.5. Let $v_{0}, v_{1}, \ldots, v_{\ell}$ be all vertices on a composite edge of $\mathscr{M}$ and $f \in \mathbf{S}(3,1 ; \mathscr{M})$. Then the Hermite data $H^{v_{i}}(f)$ depends linearly on $H^{v_{0}}(f)$ and $H^{v_{\ell}}(f)$, where $v_{i}$ is a hanging vertex, for $i=1,2, \ldots, \ell-1$.

Moreover, a hierarchical parametric mesh is T-cycle free. For a T-cycle free mesh, the Hermite data at a hanging vertex can be decided by the Hermite data at basis vertices, as [26].

Let $\mathscr{M}$ be a hierarchical mesh. The results of Sections 3.1.1, and 3.1.2 imply the following proposition.

Proposition 3.2. The map $\mathscr{H}$ defined in (3.1) can be reduced to

$$
\begin{gathered}
\widetilde{\mathscr{H}}: \mathbf{S}(3,1 ; \mathscr{M}) \rightarrow \mathbb{R}^{4 N_{1}+2 N_{2}+N_{3}}, \\
f \mapsto \oplus_{v \in V} \widetilde{H}^{v}(f)
\end{gathered}
$$

where $V$ is the set of basis vertices of $\mathscr{M}$ and

- $\widetilde{H}^{v}(f)=H^{v}(f)$, if $v$ is a boundary vertex $\operatorname{or} \operatorname{deg}(v) \bmod 4=0$,

- $\widetilde{H}^{v}(f)=\left[f(v), \frac{\partial^{2} f(v)}{\partial s \partial t}\right]$, if $\operatorname{deg}(v) \bmod 4=2$,

- $\widetilde{H}^{v}(f)=[f(v)]$, if $\operatorname{deg}(v) \bmod 2=1$.

Here, $N_{1}$ is the number of boundary vertices and interior basis vertices with $\operatorname{deg}(v)$ $\bmod 4=0, N_{2}$ is the number of interior basis vertices with $\operatorname{deg}(v) \bmod 4=2$, and $N_{3}$ is the number of interior basis vertices with $\operatorname{deg}(v) \bmod 2=1$. $\widetilde{\mathscr{H}}$ is injective.

Indeed, based on Lemma 3.5, if all Hermite data at the basis vertices vanish, then $H^{v}(f)=\mathbf{0}$ for any vertex $v$ of $\mathscr{M}$. Thus, for any cell of $\mathscr{M}$, the Hermite data of $f$ at any vertex of this cell is zero, i.e., $f \equiv 0$. In other words, $\widetilde{\mathscr{H}}$ is injective.

In the next section, we will construct splines associated with a basis vertex of a hierarchical parametric mesh $\mathscr{M}$ and prove that $\widetilde{\mathscr{H}}$ is surjective. 


\subsection{Dimension formulas and Hermite bases}

In this section, $\mathscr{M}$ is a hierarchical parametric mesh obtained by a sequence of refinements:

$$
\mathscr{M}^{0} \longrightarrow \cdots \mathscr{M}^{k} \longrightarrow \cdots \longrightarrow \mathscr{M}^{l}=\mathscr{M}
$$

We will continue to analyze $\widetilde{\mathscr{H}}$ introduced in Proposition 3.2. By proving that $\widetilde{\mathscr{H}}$ is a surjection, the dimension formulae of $\mathbf{S}(3,1 ; \mathscr{M})$ are given, and the spline set constructed in Theorem 3.2 is a basis set called Hermite bases.

As we have seen (Proposition 3.2), a spline function $f$ over $\mathscr{M}$ is uniquely determined by its Hermite data $\widetilde{\mathscr{H}}(f)$. The following lemma shows that the image of the linear map $\widetilde{\mathscr{H}}$ is the vector space of Hermite data that are compatible across each edge of $\mathscr{M}$.

Lemma 3.6. If the Hermite data at the vertices of the cells $C_{1}, \ldots, C_{N}$ are compatible, there exists a unique element $f \in \mathbf{S}(3,1 ; \mathscr{M})$ with this Hermite data.

Proof. Let us consider two vertices $v_{1}, v_{2}$ of a common edge between two cells $C_{i_{1}}, C_{i_{2}}$ of $\mathscr{M}$. We can assume that the transition map is the identity map. Let $f_{1}$ (resp. $f_{2}$ ) be the unique function constructed from the Hermite data at the vertices on $C_{1}$ (resp. $\left.C_{2}\right)$. By Lemma 3.3, $f_{1}$ and $f_{2}$ are $\mathcal{C}^{1}$ across the common edge $\left(v_{1}, v_{2}\right)$. This shows that the piecewise polynomial function $f$ constructed on each cell $C_{1}, \ldots, C_{N}$ of $\mathscr{M}$ from the Hermite data at their vertices is in $\mathbf{S}(3,1 ; \mathscr{M})$. As $\widetilde{\mathscr{H}}$ is injective, the function $f$ is uniquely determined by its Hermite data.

In the following, we are going to construct linearly independent spline functions in $\mathbf{S}(3,1 ; \mathscr{M})$. This set of spline functions is a basis of $\mathbf{S}(3,1 ; \mathscr{M})$.

To construct this basis, we proceed as follows. We will associate $J=1,2$ or 4 splines $f_{v}^{j}$ with each basis vertex $v$ of $\mathscr{M}$; the choice of $J$ follows a rule described in the theorem below. We do not associate splines with hanging vertices.

Theorem 3.2. Let $v$ be any basis vertex of a hierarchical parametric mesh $\mathscr{M}$. We can associate with $v$ a family of $J$ splines $f_{v}^{j}, j=1 \ldots J$, ( is indicated below), such that the Hermite data of each $f_{v}^{j}, j=1 \ldots J$ at all other basis vertices $w \neq v$ of $\mathscr{M}$ are 0 . While $J$ and the Hermite data of each $f_{v}^{j}$, $j=1 \ldots J$ at $v$ are as follows.

1. If $v$ is a boundary vertex or an interior vertex with $\operatorname{deg}(v) \bmod 4=0$, then $J=4$ and the Hermite data can be set equal to any one of the following choices:

$$
[1,0,0,0],[0,1,0,0],[0,0,1,0],[0,0,0,1] \text {. }
$$

2. If $v$ is an interior vertex and $\operatorname{deg}(v) \bmod 2=1,3$, then $J=1$, and the Hermite data can be set equal to $[1,0,0,0]$. 
3. If $v$ is an interior vertex and $\operatorname{deg}(v) \bmod 4=2$, then $J=2$ and the Hermite data can be set equal to any one of the following choices:

$$
[1,0,0,0],[0,0,0,1]
$$

Proof. Let us first compute the Hermite data for all of the vertices of the cells of $\mathscr{M}$.

1. Set the Hermite data for all basis vertices $w \neq v$ to zero. They naturally satisfy the constraints described in Section 3.1. Thus they are compatible.

2. Set the Hermite data at $v$ to any of the vectors mentioned in this theorem. By the constraint analysis of Section 3.1, they satisfy the compatibility condition.

3. For the Hermite data at a hanging vertex, they can be decided by the Hermite data at basis vertices of $\mathscr{M}$ as analysing after Lemma 3.5.

By Lemma 3.6, there exists a unique element of $\mathbf{S}(3,1 ; \mathscr{M})$ corresponding to these Hermite data. It satisfies the requirement of this theorem.

A direct consequence of this result is the following:

Corollary 3.3. $\widetilde{\mathscr{H}}$ is surjective.

We deduce the dimension formula for $\mathbf{S}(3,1 ; \mathscr{M})$.

Theorem 3.4 (Dimension formula). Let $\mathscr{M}$ be a hierarchical parametric mesh.

$$
\operatorname{dim} \mathbf{S}(3,1 ; \mathscr{M})=4 N_{1}+2 N_{2}+N_{3}
$$

where $N_{1}$ is the number of boundary vertices and interior basis vertices with $\operatorname{deg}(v) \bmod 4=0$, $N_{2}$ is the number of interior basis vertices with $\operatorname{deg}(v) \bmod 4=2$, and $N_{3}$ is the number of interior basis vertices with $\operatorname{deg}(v) \bmod 4=1,3$.

Remark 3.5. For $\mathbf{S}(d, r, \mathscr{M})$, we can analyze its dimension formula with the same method presented for $\mathbf{S}(3,1, \mathscr{M})$, where $d=2 r+1$.

Using the functions defined by Theorem 3.2, we get a set of bases of $\mathbf{S}(3,1 ; \mathscr{M})$ called the Hermite bases of $\mathbf{S}(3,1 ; \mathscr{M})$. In particular, we have the following property:

Corollary 3.6 (Local Support). Assume that $\mathscr{M}$ has no hanging vertices. Let $f_{v_{i}}^{1}, f_{v_{i}}^{2}, \ldots, f_{v_{i}}^{n_{i}}$ be all of the splines associated with the vertex $v_{i}$. Then, the support of each $f_{v_{i}}^{j}$ is within the 1-neighborhood of $v_{i}$.

Proof. If the mesh has no hanging vertices, the Hermite data of the basis functions in Theorem 3.2 associated with a vertex $v$ vanish at all other vertices $w \neq v$. Thus, their supports are in the union of cells of $\mathscr{M}$ containing $v$. 
Remark 3.7. The spline functions that we consider are $\mathcal{C}^{1}$-functions, after a local rigid transformation of the coordinate systems of a cell, i.e., these spline functions are $\mathcal{C}^{1}$. continuity across adjective elements from perspective of isoparametric elements method. In isoparametric elements method, the classical cubic Hermite element [3] are $\mathcal{C}^{1}$-elements. Hermite bases in this paper are a generalisation of the classical cubic Hermite elements from two aspects.

1. Local refinement (Section 2.2.3). This property allows us to use adaptive mesh refinement strategies.

2. Complex topology: Hermite bases defined on a mesh with complex topology. This enables us to align complex iso-curves. However, to maintain $\mathcal{C}^{1}$-continuity at the interior extraordinary vertices, by Proposition 3.1, the degrees of freedom of the classical cubic Hermite elements are lost, i.e., Hermite bases at the interior extraordinary vertices are incomplete. This problem is solved by setting a proper parameterization (see $\mathbf{R}_{2}$ of Section 4.1) and Theorem 4.2.

\section{Parameterizations and the interpolation approximation error}

In this section, we analyze the spline spaces from an approximation perspective. A sketch of the parameterization algorithm is given in Section 4.1 for analyzing the requirements of constructing parameterization. The parameterization of a physical domain is represented in a linear combination of the Hermite bases constructed in Section 3.2. Using this construction, we analyze in Section 4.2 the approximation error order of the spline spaces using the Hermite basis representation.

\subsection{Parameterization}

There are three steps for generating a parameterization $\mathscr{P}$ that aligns the principal directions of the transport process, i.e., iso-curves of the magnetic flux surfaces defined on a given physical domain.

- Based on the iso-curves, design a parametric mesh $\mathscr{M}$ such that these iso-curves can be treated as the image of some mesh grid lines of $\mathscr{M}$ under a parameterization $\mathscr{P}$;

- Choose positions $\mathbf{Q}=\left\{Q_{i}\right\}$ on the physical domain and their parameters $\mathbf{P}=\left\{P_{i}\right\}$ with frame $\mathscr{F}$, where we expect $\mathscr{P}\left(P_{i}\right)=Q_{i}$;

- Minimize the energy $E$ :

$$
E=\sum_{P_{i} \in \mathbf{P}} \omega_{i}\left\|\mathscr{P}\left(P_{i}\right)-Q_{i}\right\|^{2}
$$

where $\left\{\omega_{i}\right\}$ are weights. 
Then, a parameterization $\mathscr{P}: \mathscr{M} \longrightarrow \Omega$ is obtained, where $\Omega$ is a physical domain. The choice of weights follows a rule implying that the iso-curves in the physical domain can be aligned by mesh grid lines.
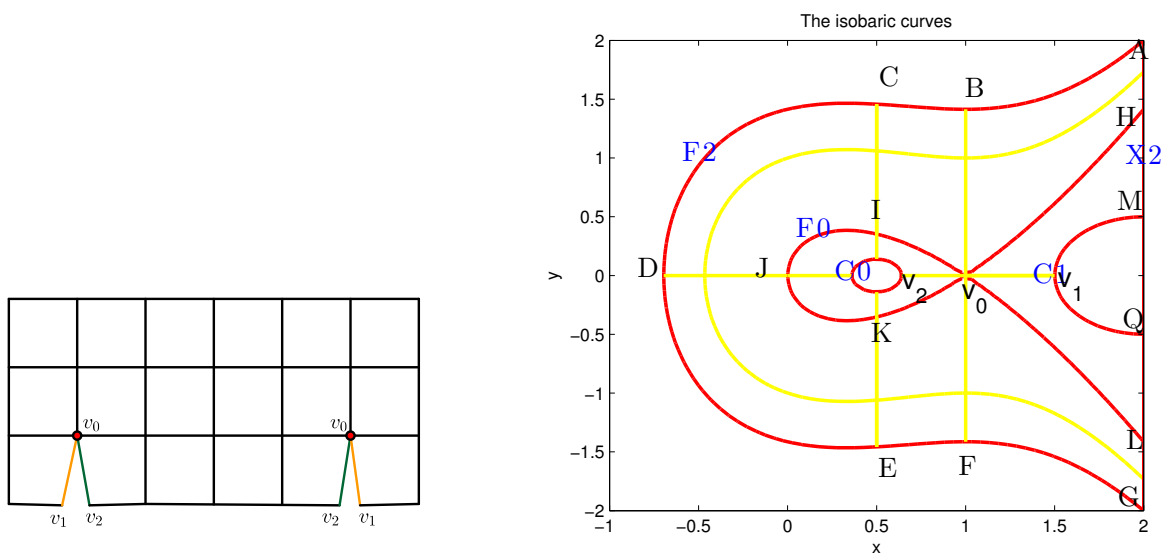

Figure 7: Parametric mesh of Example 4.1, where $\operatorname{deg} v_{0}=8$

In the following example, the topology of the parametric mesh is the same as that of the mesh of the target application.

\section{Example 4.1.}

The physical domain $\Omega$ shown in the left of Figure 8 is bounded by $y^{2}-x(x-1)^{2}=$ $2,(x-2)^{2}+y^{2}=0.25, x=2,(x-0.5)^{2}+y^{2}=(\sqrt{2} / 10)^{2}$. The iso-curves are given by $F 0$ : $y^{2}-x(x-1)^{2}=0, F 2: y^{2}-x(x-1)^{2}=2, X 2: x=2, C 0:(x-0.5)^{2}+y^{2}=(\sqrt{2} / 10)^{2}$ and $C 1:$ $(x-2)^{2}+y^{2}=0.25$. Then we construct a parametric mesh $\mathscr{M}$ shown in the left of Figure 7. Some grid lines of $\mathscr{M}$ are expected to align the iso-curves shown on the right of Figure 7.

Take $\mathbf{Q}=\left\{Q_{i}\right\}$ in $\Omega$ and $\mathbf{P}=\left\{P_{i}\right\}$ on $\mathscr{M}$, where $\mathbf{Q}$ is shown in the middle of Figure 8. Then, minimize the energy (4.1) with weights of 1.5 for the points on the iso-curves and weights of 1.0 for the other points. The obtained parameterization $\mathscr{P}$ is shown on the right of Figure 8. Based on Example 4.1, Hermite bases can be used to represent the physical domain with complex iso-curves. However, even if there are 4 degrees of freedom at $v_{0}$ in Figure 7, there is only one degree of freedom used by the result of this algorithm.

Notation 4.1. In the following, parameterizations should satisfy:

1. At the extraordinary vertices, only one Hermite basis corresponding to $[1,0,0,0]$ is used to construct parameterizations. There are two reasons:

$\mathbf{R}_{1}$ : Avoid a geometric foldover of a physical domain.

$\mathbf{R}_{2}$ : Obtain the optimal approximation order: As mentioned in the second point of Remark 3.7, Hermite bases at the interior extraordinary vertices are incomplete. With only 

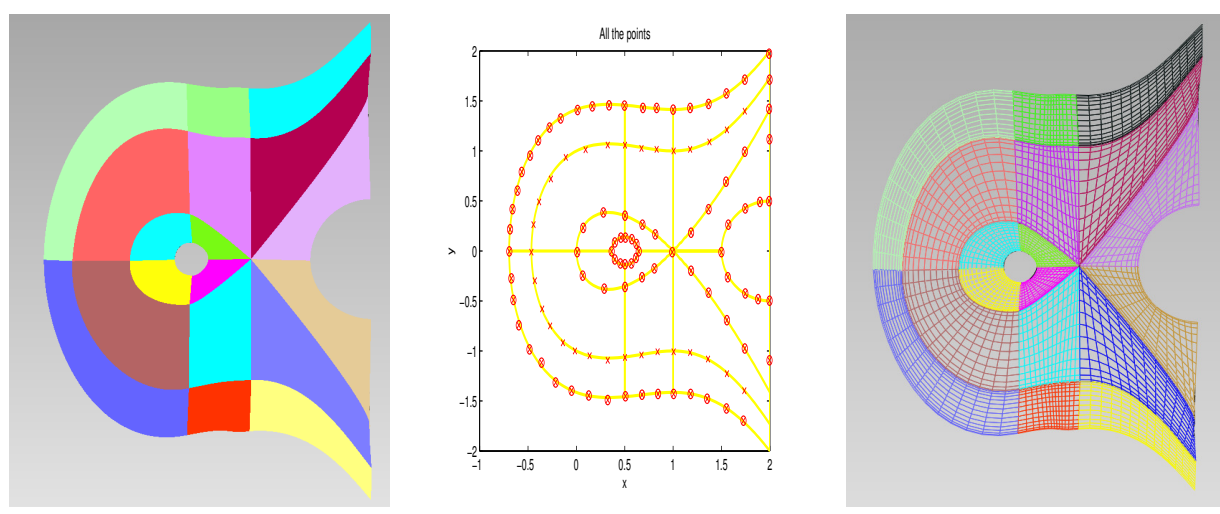

Figure 8: Physical domain (left), $\mathbf{Q}=\left\{Q_{i}\right\}$ (middle) and parameterization of the physical domain(right)

one Hermite basis corresponding to $[1,0,0,0]$, we can reduce the impact of incompleteness on physical domains (see Case 2 in the proof of Theorem 4.2, $\mathscr{J} \sim h^{2}$ ) and obtain the optimal interpolation approximation order.

2. For solving PDEs, integrability assumptions should be considered, if there is an extraordinary vertex. In the examples of Section 5, parameterizations of physical domains are designed such that they satisfy the $H^{1}$ integrability assumptions in [27] for sloving the Grad-Shafanov equation which is a second order PDE.

\subsection{The interpolation approximation error by bicubic Hermite bases}

In this section, we analyze the convergence behavior of Hermite bases and the approximation error by bicubic Hermite bases. We show that the optimal approximation rate is reached.

Let $\mathscr{M}_{0}$ be an initial parametric mesh. When we subdivide its cells into 4 sub-cells, i.e., subdivide one time in each direction, the extraordinary vertices will be surrounded by the vertices whose valences are 4 , and the number of extraordinary vertices will not change. For example, the topology of the initial parametric mesh is shown on the left side of Figure 9. The topology of the parametric mesh after one subdivision step is shown on the right handside of Figure 9. The number of extraordinary vertices and the number of cells with an extraordinary vertex as a corner vertex do not change in the subdivision process. Let $v$ be an extraordinary vertex of the initial parametric mesh $\mathscr{M}_{0}$. After subdividing $k$ times uniformly, we obtain the parametric mesh $\mathscr{M}_{k}$. There are two classes of cells of $\mathscr{M}_{k}$. The first is a cell with 4 regular vertices, i.e., all of the vertices are boundary vertices, or their valences are 4 , the second is a cell with one extraordinary vertex as its vertex.

Theorem 4.2. Let $\mathscr{M}_{0}$ be a parametric mesh with cells of size 1 and with at most one extraordinary vertex per cell. Let $\mathscr{M}_{k}$ be the parametric mesh obtained by subdividing $\mathscr{M}_{0} k$ times uniformly with cells of size $h=2^{-k}$. Let $\mathscr{P}: \mathscr{M}_{0} \longrightarrow \Omega$ be a bijective parameterization represented in the Hermite basis over $\mathscr{M}_{0}$. Suppose that $F(x, y) \in \mathcal{C}^{4}(\Omega)$. There exists $K \in \mathbb{R}_{+}$, such that $\forall k \in \mathbb{N}$, 

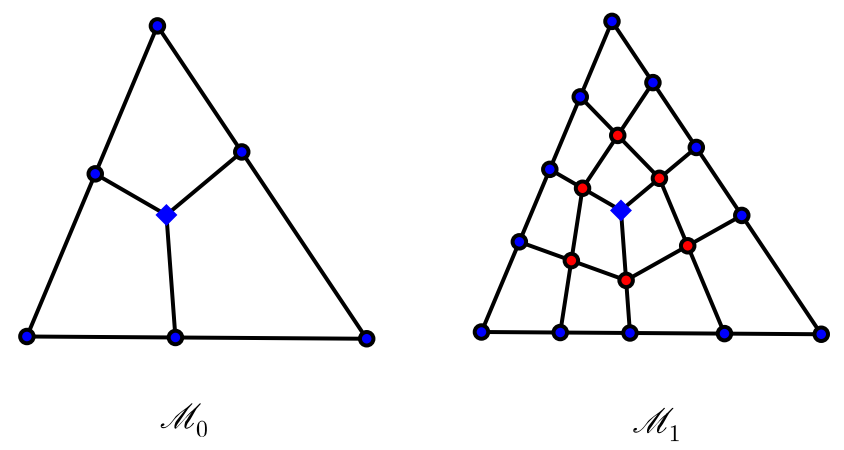

Figure 9: Subdivide the initial parametric mesh $\mathscr{M}_{0}$

there exists $u \in \mathbf{S}\left(3,1 ; \mathscr{M}_{k}\right)$ such that

$$
\left\|F(x, y)-u \circ \mathscr{P}^{-1}\right\|_{L^{2}} \leq K h^{4} .
$$

Proof. First, we construct $u \in \mathbf{S}\left(3,1 ; \mathscr{M}_{k}\right)$. For each vertex $v_{i}$ of $\mathscr{M}_{k}, F \circ \mathscr{P}^{\prime}$ s Hermite data can be used to recover a spline defined over $\mathscr{M}_{k}$. To be more specific, let $C=\left[s_{1}, s_{1}+h\right] \times$ $\left[t_{1}, t_{1}+h\right]$ be a cell with $v_{i}$ as its vertex, where $h=2^{-k}$.

- If $v_{i}$ 's valence is 4 or $v_{i}$ is a boundary vertex, i.e. $v_{i}$ is regular, the coefficients of the 4 Hermite bases can be obtained by

$$
H^{v_{i}}(F \circ \mathscr{P})=\left[\left.F \circ \mathscr{P}\right|_{C}\left(v_{i}\right), \frac{\left.\partial F \circ \mathscr{P}\right|_{C}}{\partial s}\left(v_{i}\right), \frac{\left.\partial F \circ \mathscr{P}\right|_{C}}{\partial t}\left(v_{i}\right), \frac{\left.\partial^{2} F \circ \mathscr{P}\right|_{C}}{\partial s \partial t}\left(v_{i}\right)\right],
$$

where $(s, t)$ are the coordinates associated with $C$;

- Otherwise, there is only one Hermite basis function attached to $v$ with Hermite data $[1,0,0,0]$. Its coefficient is

$$
H^{v_{i}}(F \circ \mathscr{P})=\left[F \circ \mathscr{P}\left(v_{i}\right)\right]
$$

Thus, we define a spline function $u$ over $\mathscr{M}_{k}$ as the linear combination of the Hermite basis with coefficients corresponding to these values:

$$
u=\sum_{v} H^{v}(F \circ \mathscr{P}) \cdot \phi_{v}
$$


where $\phi_{v}$ is the vector of Hermite basis functions attached to the vertex $v$ and $H^{v}(F \circ \mathscr{P})$ is the Hermite data of the function $F \circ \mathscr{P}$ at the vertex $v$.

Let $U=u \circ \mathscr{P}^{-1}$. We estimate the $L^{2}$ error

$$
\begin{aligned}
& \|U(x, y)-F(x, y)\|_{L^{2}}^{2}=\int_{\Omega}(U(x, y)-F(x, y))^{2} d x d y \\
& \quad=\sum_{C_{i}^{1}} \int_{\mathscr{P}\left(C_{i}^{1}\right)}(U(x, y)-F(x, y))^{2} d x d y+\sum_{C_{i}^{2}} \int_{\mathscr{P}\left(C_{i}^{2}\right)}(U(x, y)-F(x, y))^{2} d x d y
\end{aligned}
$$

where $C_{i}^{1}$ is a cell with 4 regular vertices and $C_{i}^{2}$ is a cell with one extraordinary vertex as its vertex. We will estimate $\int_{\mathscr{P}\left(C_{i}^{1}\right)}(U(x, y)-F(x, y))^{2} d x d y$ and $\int_{\mathscr{P}\left(C_{i}^{2}\right)}(U(x, y)-$ $F(x, y))^{2} d x d y$, respectively.

Case 1: There are 4 Hermite bases at each regular vertex of the cell $C_{i}^{1}=\left[s_{1}, s_{1}+h\right] \times\left[t_{1}, t_{1}+\right.$ $h]$ of $\mathscr{M}_{k}$. Then $\left.u\right|_{C_{i}^{1}}$ s Hermite data at each vertex of $C_{i}^{1}$ is the same as the Hermite data of $\left.F \circ \mathscr{P}\right|_{C_{i}^{1}}$ at these vertices. Because $F(x, y) \in \mathcal{C}^{4}(\Omega)$ and each component of $\left.\mathscr{P}\right|_{C_{i}^{1}}$ is a bicubic polynomial. $\left.F \circ \mathscr{P}\right|_{C_{i}^{1}} \in \mathcal{C}^{4}\left(C_{i}^{1}\right)$. By Theorem 4 in [29], $\forall(s, t) \in C_{i}^{1}$

$$
|F \circ \mathscr{P}|_{C_{i}^{1}}(s, t)-\left.\left.u\right|_{C_{i}^{1}}(s, t)\right|_{\infty} \leq K_{1} h^{4},
$$

where $K_{1} \in \mathbb{R}_{+}$is a constant depending only on $F \circ \mathscr{P}$. Thus,

$$
\int_{\mathscr{P}\left(C_{i}^{1}\right)}(F(x, y)-U(x, y))^{2} d x d y \leq \int_{C_{i}^{1}}\left(\left.F \circ \mathscr{P}\right|_{C_{i}^{1}}(s, t)-\left.u\right|_{C_{i}^{1}}(s, t)\right)^{2}\left|\mathscr{J}_{C_{i}^{1}}(s, t)\right| d s d t
$$

where

$$
\mathscr{J}_{C_{i}^{1}}(s, t)=\left|\begin{array}{ll}
\frac{\partial x(s, t)}{\partial s} & \frac{\partial x(s, t)}{\partial t} \\
\frac{\partial y(s, t)}{\partial s} & \frac{\partial y(s, t)}{\partial t}
\end{array}\right|
$$

with $\left.\mathscr{P}\right|_{C_{i}^{1}}(s, t)=[x(s, t), y(s, t)]$. There is a constant $K_{2} \in \mathbb{R}_{+}$depending only on $\mathscr{P}$, such that $\forall(s, t) \in C_{i}^{1}$

$$
\left|\mathscr{J}_{C_{i}^{1}}(s, t)\right| \leq K_{2}
$$

Thus

$$
\begin{aligned}
\int_{\mathscr{P}\left(C_{i}^{1}\right)}(F(x, y)-U(x, y))^{2} d x d y & \leq K_{1} K_{2}\left(h^{4}\right)^{2} \int_{C_{i}^{1}} d s d t \\
& =K_{1} K_{2} h^{10},
\end{aligned}
$$

The number of cells of this type in $\mathscr{M}_{k+1}$ is less than $N_{0}\left(2^{k}\right)^{2}=N_{0} / h^{2}$, where $N_{0}$ is the number of cells of $\mathscr{M}_{0}$. Thus,

$$
\begin{aligned}
\sum_{C_{i}^{1}} \int_{\mathscr{P}\left(C_{i}^{1}\right)}(U(x, y)-F(x, y))^{2} d x d y & \leq K_{1} K_{2} h^{10}\left(N_{0} / h^{2}\right) \\
& =K_{1} K_{2} N_{0} h^{8},
\end{aligned}
$$


Case 2: Let $C_{i}^{2}$ be a cell with one extraordinary vertex $v_{4}$ and the other three vertices $v_{1}, v_{2}, v_{3}$ of $C_{i}^{2}$ of valence 4 . Suppose $C_{i}^{2}=\left[s_{1}, s_{1}+h\right] \times\left[t_{1}, t_{1}+h\right], v_{1}=\left(s_{1}, t_{1}\right), v_{2}=\left(s_{1}+\right.$ $\left.h, t_{1}\right), v_{3}=\left(s_{1}, t_{1}+h\right), v_{4}=\left(s_{1}+h, t_{1}+h\right)$. Then the spline $u$ defined over $\mathscr{M}_{k}$ satisfies

- $u\left(v_{4}\right)=F \circ \mathscr{P}\left(v_{4}\right)$;

- $H_{(s, t)}^{v_{i}}(u)=H_{(s, t)}^{v_{i}}(F \circ \mathscr{P})$, i. e. ,

$$
u\left(v_{i}\right)=F \circ \mathscr{P}\left(v_{i}\right) ; \frac{\partial u\left(v_{i}\right)}{\partial s}=\frac{\partial F \circ \mathscr{P}\left(v_{i}\right)}{\partial s} ; \frac{\partial u\left(v_{i}\right)}{\partial t}=\frac{\partial F \circ \mathscr{P}\left(v_{i}\right)}{\partial t} ; \frac{\partial^{2} u\left(v_{i}\right)}{\partial s \partial t}=\frac{\partial^{2} F \circ \mathscr{P}\left(v_{i}\right)}{\partial s \partial t},
$$

where $i=1,2,3$.

Let $\rho(s, t)=\left.F \circ \mathscr{P}\right|_{C_{i}^{2}}(s, t)-\left.u\right|_{C_{i}^{2}}(s, t)=\left.F \circ \mathscr{P}\right|_{C_{i}^{2}}(s, t)-\left.\sum_{i=1}^{4} H^{v_{i}}(F \circ \mathscr{P}) \cdot \phi_{v_{i}}\right|_{C_{i}^{2}}(s, t)$. There is a constant $K_{3} \in \mathbb{R}$ depending only on $F \circ \mathscr{P}$ such that $\forall(s, t) \in C_{i}^{2}$,

$$
\left|\frac{\partial^{2} \rho}{\partial u \partial v}(s, t)\right| \leq\left|\frac{\partial^{2} F \circ \mathscr{P}}{\partial u \partial v}(s, t)\right| \leq \sum_{i=1}^{4}\left|H^{v_{i}}(F \circ \mathscr{P}) \cdot \frac{\partial^{2} \phi_{v_{i}}}{\partial u \partial v}(s, t)\right| \leq K_{3}
$$

for $\partial u, \partial v \in\{\partial s, \partial t\}$.

By construction,

$$
\rho\left(v_{i}\right)=0, \frac{\partial \rho}{\partial s}\left(v_{i}\right)=0, \frac{\partial \rho}{\partial t}\left(v_{i}\right)=0 .
$$

for $i=1, \ldots, 3$. Thus, by Taylor expansion at $v_{1}$, we have for all $p=(s, t) \in C_{i}^{2}$ there exist $q \in C_{i}^{2}$ such that

$$
\rho(p)=\frac{1}{2} \frac{\partial^{2} \rho}{\partial \mathbf{l}^{2}}(q)\left|v_{1} p\right|^{2}=\frac{1}{2}\left(l_{1}^{2} \frac{\partial^{2} \rho}{\partial s^{2}}(q)+2 l_{1}, l_{2} \frac{\partial^{2} \rho}{\partial s \partial t}(q)+l_{2}^{2} \frac{\partial^{2} \rho}{\partial t^{2}}(q)\right)\left|v_{1} p\right|^{2}
$$

where $\mathbf{l}=\overrightarrow{v_{1}} p /\left|\overrightarrow{v_{1}} p\right|$. We deduce that

$$
\left|\frac{\partial^{2} \rho}{\partial \mathbf{l}^{2}}(p)\right| \leq K_{3}\left|v_{1} p\right|^{2} \leq 2 K_{3} h^{2} .
$$

Let $\left.\mathscr{P}\right|_{C_{i}^{2}}(s, t)=[x(s, t), y(s, t)]$. The parameterization $\mathscr{P}$ is singular at $v_{4}$, i.e.,

$$
\begin{aligned}
& \frac{\partial x\left(v_{4}\right)}{\partial s}=0, \frac{\partial x\left(v_{4}\right)}{\partial t}=0 ; \\
& \frac{\partial y\left(v_{4}\right)}{\partial s}=0, \frac{\partial y\left(v_{4}\right)}{\partial t}=0 .
\end{aligned}
$$

By Taylor expansion of its Jacobian

$$
\mathscr{J}_{C_{i}^{2}}(s, t)=\left|\begin{array}{ll}
\frac{\partial x(s, t)}{\partial s} & \frac{\partial x(s, t)}{\partial t} \\
\frac{\partial y(s, t)}{\partial s} & \frac{\partial y(s, t)}{\partial t}
\end{array}\right|
$$


at the extraordinary vertex $v_{4}$, we have $\forall(s, t) \in C_{i}^{2}$

$$
\left|\mathscr{J}_{C_{i}^{2}}(s, t)\right| \leq K_{4} h^{2}
$$

for some $K_{4} \in \mathbb{R}$ depending only on $\mathscr{P}$. We deduce that

$$
\begin{aligned}
\int_{\mathscr{P}\left(C_{i}^{2}\right)}(U(x, y)-F(x, y))^{2} d x d y & =\int_{C_{i}^{2}}\left(\left.F \circ \mathscr{P}\right|_{C_{i}^{2}}(s, t)-\left.u\right|_{C_{i}^{2}}(s, t)\right)^{2}\left|\mathscr{J}_{C_{i}^{2}}(s, t)\right| d s d t \\
& \leq 2 K_{3} K_{4} h^{6} \int_{C_{i}^{2}} d s d t=2 K_{3} K_{4} h^{8} .
\end{aligned}
$$

The number of cells of the second type of cells does not change in the subdivision process, i.e., it is a positive constant, denoted $N_{i r}$, for each $\mathscr{M}_{k}$. Thus, there is a positive constant $K_{5}$ such that

$$
\sum_{C_{i}^{2}} \int_{\mathscr{P}\left(C_{i}^{2}\right)}(U(x, y)-F(x, y))^{2} d x d y \leq 2 N_{i r} K_{3} K_{4} h^{8}=K_{5} h^{8}
$$

Based on (4.4) and (4.5), there is a positive constant $K_{6}$ depending only on $\mathscr{M}_{0}, \mathscr{P}$ and $F$, such that

$$
\begin{aligned}
& \int_{\Omega}(F(x, y)-U(x, y))^{2} d x d y \\
& =\sum_{C_{i}^{1}} \int_{\mathscr{P}\left(C_{i}^{1}\right)}(F(x, y)-U(x, y))^{2} d x d y+\sum_{C_{i}^{2}} \int_{\mathscr{P}\left(C_{i}^{2}\right)}(F(x, y)-U(x, y))^{2} d x d y \\
& \leq K_{6} h^{8} .
\end{aligned}
$$

By (4.6), for $F(x, y) \in \mathcal{C}^{4}(\Omega)$, there is a spline $u$ defined over $\mathscr{M}_{k}$ such that

$$
\left\|F(x, y)-u \circ \mathscr{P}^{-1}\right\|_{L^{2}}=\sqrt{\int_{\Omega}\left(F(x, y)-u \circ \mathscr{P}^{-1}\right)^{2} d x d y} \leq K h^{4},
$$

where $K=\sqrt{K_{6}} \in \mathbb{R}$ depends only on $\mathscr{M}_{0}, F$ and $\mathscr{P}$. In other words, for a smooth function $F(x, y)$ defined over the physical domain, there is a spline $u$ defined over $\mathscr{M}_{k}$ that can be used to approximate $F(x, y)$ by an injective parameterization $\mathscr{P}$ with fourth order of approximation for the $L_{2}$ norm.

Remark 4.1. In the context of Isogeometric analysis with Galerkin formulation using this type of splines, a projection of the solution of the PDE on the space of functions of the form $u \circ \mathscr{P}^{-1}$ with $u \in \mathbf{S}\left(3,1 ; \mathscr{M}_{k}\right)$ is computed. The order of approximation of the projected solutions, which minimizes the $L_{2}$ distance to this space, should be at least 4 . 


\section{$5 \quad$ Numerical convergence rates for bicubic Hermite bases}

In classical FEM error analysis (see, e.g., Theorem 6.2.1 and Proposition 6.2.2 in [28]), if the exact solution is in $H^{4}(\Omega)$, it is expected that the approximation order with bicubic splines test functions is 4 for the $L^{2}$-norm and 3 for the $H^{1}$-norm. In this section, the Hermite bases constructed in Section 3.2 are used to represent the parameterizations of physical domains, and they are treated as trail and test functions when solving the PDE. The numerical examples for solving the Grad-shafanov equation show that the numerical convergence rates measured by $L^{2}$-norm and $H^{1}$-norm are as expected. The reasons for choosing these examples are the following.

1. The physical domain can be exactly described by the parameterization with Hermite bases. The error between the exact solution and the solution given by the isoparametric finite element method or isogeometric analysis comes from two parts. One is the approximation error of the trail functions. The other is the approximation error of the physical domain by the shape functions. Thus, to test the error orders that come from the approximation of our spline spaces of solving PDEs, we choose physical domains that can be exactly described by Hermite bases.

2. The Grad-Shafanov equation [30] is chosen as PDE for describing the resitive MHD problem. Let $\Omega$ be a physical domain. The generic form of the fixed-boundary GradShafranov equation can be written as

$$
\begin{aligned}
& \triangle^{*} u=f_{0}(u, r, z) \text { in } \Omega \\
& u=0 \text { on } \partial \Omega
\end{aligned}
$$

where

$$
\begin{aligned}
\triangle^{*} u & =r \frac{\partial}{\partial r}\left(\frac{1}{r} \frac{\partial u}{\partial r}\right)+\frac{\partial^{2} u}{\partial z^{2}} \\
& =r \nabla(X \nabla u),
\end{aligned}
$$

and

$$
X=\left(\begin{array}{cc}
1 / r & 0 \\
0 & 1 / r
\end{array}\right)
$$

Thus, in the following examples, we consider

$$
\begin{aligned}
-\nabla(R(r) \nabla u) & =-g(r) f(u, r, z) \text { in } \Omega, \\
u & =0 \text { on } \partial \Omega,
\end{aligned}
$$

where $g(r) \in L^{2}(\Omega)$ is a function of $r$ and

$$
R(r)=\left(\begin{array}{cc}
g(r) & 0 \\
0 & g(r)
\end{array}\right)
$$


Case 1. If $f(u, r, z)=f(r, z) \in L^{2}(\Omega)$, i.e., $f(u, r, z)$ has no relationship with $u$, then the weak form of this model PDE (5.2) can be stated as follows. Given $f$, find $u \in V$, such that for all $v \in V$,

$$
a(u, v)=l(v),
$$

where $V=\left\{v: v \in \mathbf{H}^{1}(\Omega),\left.v\right|_{\partial \Omega}=0\right\}, \mathbf{H}^{1}(\Omega)$ is the Sobolev space that consists of the functions in $\mathbf{L}^{2}(\Omega)$ that possess weak and square-integrable derivatives. $a(u, v)$ is the symmetric bilinear form defined as

$$
a(u, v)=\int_{\Omega} \nabla v^{T} R(r) \nabla u \mathrm{~d} \Omega,
$$

$l(v)$ is a linear continuous functional defined as

$$
l(v)=-\int_{\Omega} g(r) f(r, z) v \mathrm{~d} \Omega .
$$

We discretize the weak form, Eq. (5.3), with our splines. This yields the linear system

$$
\operatorname{Ad}=\mathbf{F},
$$

where $\mathbf{A}$ is the stiffness matrix, $\mathbf{F}$ is the force vector and $\mathbf{d}$ is the displacement vector.

Case 2. For a general $f(u, r, z)$ such that (5.2) is a nonlinear Grad-Shafranov equation, the fixed-point iteration method [32] is used to compute the solution. Take $u_{0}(r, z)$ as an initial solution. Suppose that the $i-$ th iteration solution $u_{i}(r, z)$ has been obtained. Solve the $(i+1)$-th iteration solution $u_{i+1}(r, z)$ with

$$
\begin{aligned}
-\nabla\left(R(r) \nabla u_{i+1}(r, z)\right) & =-g(r) f\left(u_{i}(r, z), r, z\right) \text { in } \Omega, \\
u_{i+1} & =0 \text { on } \partial \Omega,
\end{aligned}
$$

by the method presented in Case 1. Then the solution of model PDE (5.2) is given by

$$
u(r, z)=\lim _{i \rightarrow \infty} u_{i}(r, z)
$$

3. Extraordinary vertices of the initial parametric meshes of these examples are isolated, while the parametric mesh is subdivided. The parameterizations constructed in Section 4.1 satisfy the $H^{1}$ integrability assumption [27], i.e., test functions, by composing with the inverse of a parameterization with Hermite bases, are $H^{1}$ on the physical domain.

In the following, we present two examples of parameterization with extraordinary points and show the results of numerical experimentation for the solution of Grad-Shafranov equations. All of the physical domains of these examples can be exactly described by Hermite bases. Thus, the errors come from approximation by Hermite bases. In Example 5.1, we take $g(r) \equiv 1$ and $f(u, r, z)=f(r, z)$ in the model PDE (5.2). In Example 5.2, $g(r)=1 /(r+2)^{2}$ and $f(u, r, z)=f(r, z)+u^{2}$, where $f(r, z)$ will be defined in Example 5.2. The exact solutions in Examples 5.1 and 5.2 are $\mathcal{C}^{4}(\Omega)$. 

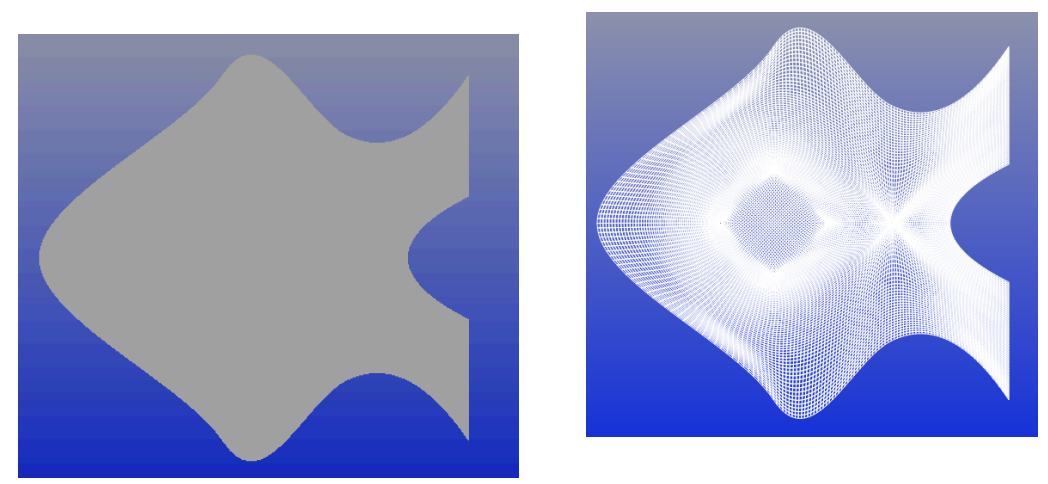

Figure 10: The physical domain and the parameterization of Example 5.1

\section{Example 5.1.}

The physical domain is shown on the left side of Figure 10, and its boundary is composed of the solutions of nine implicit bicubic polynomials, i. e., there are $F_{1}(r, z), F_{2}(r, z), \cdots, F_{9}(r, z)$, which are bicubic polynomials, such that the boundary of this physical domain is a part of the solution of one of these equations: $F_{1}(r, z)=0, F_{2}(r, z)=0, \ldots, F_{9}(r, z)=0$ The initial parametric mesh has the same topology as the "usual" mesh shown in Figure 11.

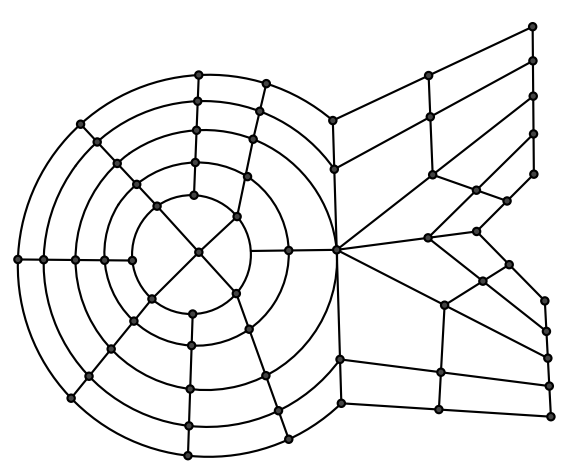

Figure 11: The "usual" mesh that has the same topology as the initial parametric mesh of Example 5.1

We take $g(r) \equiv 1$, and $f(r, z)=-\Delta u^{*}$ where $u^{*}=(r+2)(r+1) \Pi_{i}^{9} F_{i}(r, z) / 10000$. Then $u^{*}$ is the exact solution of the PDE (5.2)

$$
\begin{aligned}
& -\Delta u=f \text { in } \Omega ; \\
& u=0 \text { on } \partial \Omega .
\end{aligned}
$$

The initial parameterization of the physical domain is shown on the right side of Figure 10 with the exact boundary representation of the physical domain; 
We globally refine the spline space by recursively splitting each of the original cells of the mesh into four subcells. Figure 12 shows the numerical solution when $k=8$ and Figure 13 shows the errors measured with $L^{2}$-norm and $H^{1}$-norm.

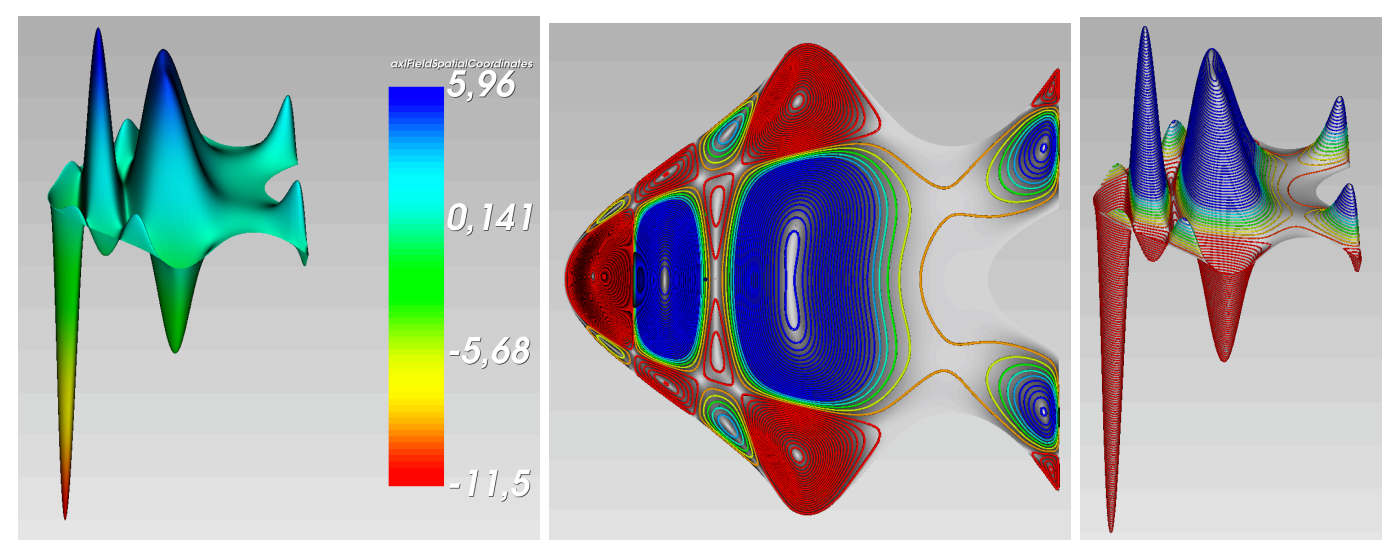

Figure 12: The numerical solution when $k=8$

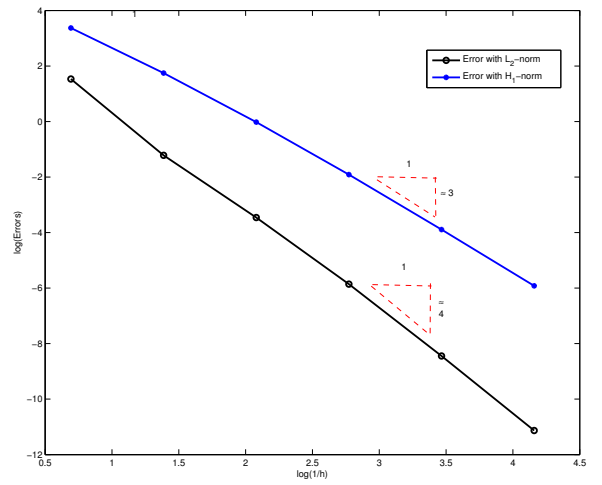

Figure 13: Errors with the $L^{2}$-norm and $H^{1}$-norm

\section{Example 5.2.}

The physical domain is the square $\Omega=[-1,1] \times[-1,1] \subset \mathbb{R}^{2}$;

We take $g(r)=1 /(r+2)^{2} f(u, r, z)=G(r, z)+u^{2}$ so that the exact solution of the model PDE (5.2) is $u=\left(1-r^{2}\right)\left(1-z^{2}\right)$, where $G(r, z)=-\left(1-r^{2}\right)^{2}\left(1-z^{2}\right)^{2}+2\left(1-z^{2}\right)-8\left(1-z^{2}\right) /(r+2)-$ $2\left(1-r^{2}\right)$. Under these conditions, the PDE is

$$
\begin{aligned}
-(r+2)^{2} \nabla(R(r) \nabla u) & =-G(r, z)-u^{2} \text { in } \Omega, \\
u & =0 \text { on } \partial \Omega,
\end{aligned}
$$


where

$$
R(r)=\left(\begin{array}{cc}
1 /(r+2)^{2} & 0 \\
0 & 1 /(r+2)^{2}
\end{array}\right)
$$

The initial parametric mesh has the same topology as the "usual" mesh shown in Figure 14;

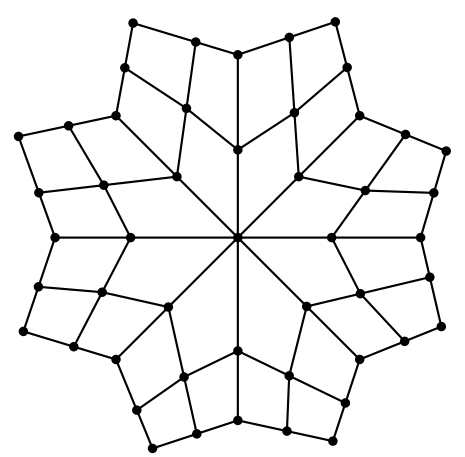

Figure 14: The "usual" mesh that has the same topology as the initial parametric mesh of Example 5.2

The parameterization of $\Omega$ (with an exact boundary representation) is described in Figure 15.
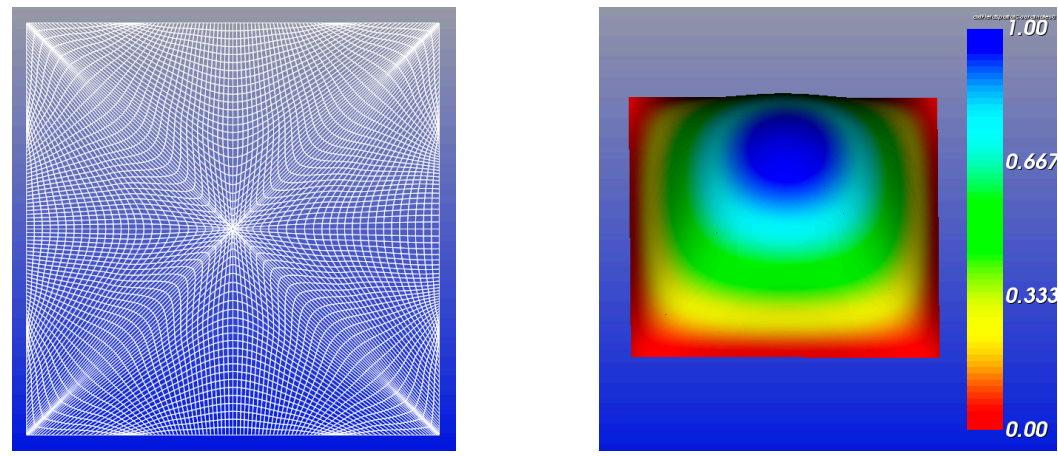

Figure 15: The parameterization of $\Omega$ and the isogeometric solution

We globally refine the spline space by recursively splitting each of the 8 cells of the mesh into four subcells. In Figure 16, there are errors measured by $L^{2}$-norm and $H^{1}$-norm.

For Examples 5.1 and 5.2, the error order with $L^{2}$-norm is approximately 4 and that with $H^{1}$-norm is approximately 3 . This means that the optimal error orders are reached in these examples. 


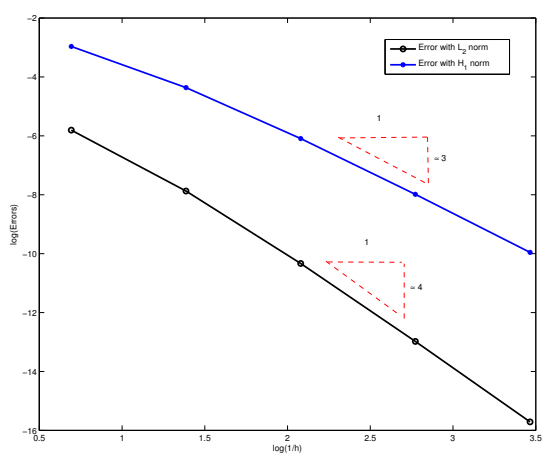

Figure 16: Errors with the $L^{2}$-norm and $H^{1}$-norm

\section{Conclusion and future work}

This paper presents the definition of spline spaces over rectangular meshes with complex topological structures. A rule for local refinement of parametric meshes is presented, and the changes in spline space over these refined parametric meshes are studied by taking transition maps as rigid transformations. Especially, the property of bi-cubic spline spaces with $\mathcal{C}^{1}$ parameter continuity is studied in detail for using it to solve PDE problems over a physical domain with a general topology. We compute their dimension and construct an explicit set of basis functions called Hermite bases. Besides the definition of splines, we obtain the following other interesting properties for solving PDEs. For instance, Theorem 4.2 presents the $L^{2}$ approximation error by Hermite bases. The error order is optimal. Moreover, the numerical convergence rates for solving the linear and nonlinear Grad-Shafranov equation are analyzed. In the numerical experiments, optimal convergence rates with $L^{2}$-norm and $H^{1}$-norm are reached.

For a better solution of the MHD simulation over a general physical domain, further investigations are considered in the following directions:

- We need to generate a parameterization by Hermite bases that satisfes integrability assumptions, improving the quality of stiffness matrix;

- A parameterization algorithm should be developed for a better approximation of the boundaries of physical domains during the subdivision process.

\section{Acknowledgments}

The authors are grateful to the anonymous reviewers for their useful comments and suggestions. Authors are supported by the National French Research Program (ANR): ANEMOS (2011), ANR-11-MONU-002. The first author is also supported by National 
Natural Science Foundation of China (Grant No.11526069), the Anhui Provincial Natural Science Foundation (No. 1608085QA14), and the Open Project Program of the State Key Lab of CAD\&CG (Grant No. A1601), Zhejiang University.

\section{References}

[1] O. Czarny and G. Huysmans. Bézier surfaces and finite elements for MHD simulations. Journal of Computational Physics, vol. 227, p. 7423-7445, 2008.

[2] G. T. A. Huysmans, J. P. Goedbloed. "Isoparametric Bicubic Hermite Elements for Solution of the Grad-Shafranov Equation". International Journal of Modern Physics C, p. 371-376, 1991.

[3] K. H. Huebner, E. A. Thornton. The Finite Element Method for Engineers. John Wiley \& Sons, ISBN 0-471-09159-6, 1982.

[4] K. Höllig. Finite Element Methods with B-Splines. SIAM, 2003.

[5] D. R. Forsey and R. H. Bartels. Hierarchical B-spline refinement. In Proceedings of the 15th annual conference on Computer graphics and interactive techniques, SIGGRAPH '88, pages 205212, New York, NY, USA, 1988. ACM.

[6] R. Kraft, Adaptive and linearly independent multilevel B-splines. In Surface Fitting and Multiresolution Methods, A. L. Méhauté, C. Rabut, and L. L. Schumaker, Eds., vol. 2. Vanderbilt University Press, p. 209 - 216.

[7] C. Giannelli, B. Jüttler, and H. Speleers. THB-splines: The truncated basis for hierarchical splines. Computer Aided Geometric Design, 29(7):485 - 498, 2012.

[8] T. Dokken, T. Lyche, and K. Pettersen. Polynomial splines over locally refined box-partitions. Computer Aided Geometric Design, 30(3), p. 331-356, 2013.

[9] T. W. Sederberg, J. Zheng, A. Bakenov, A. Nasri. T-splines and T-NURCCs. ACM Trans. Graph., vol. 22, p. 161-172, 2003.

[10] I. Ergatoudis, B. Irons, and O. Zienkiewicz. Curved isoparametric "quadrilateral" elements for finite element analysis. Int. J. Solids Structures, vol. 4, p. 31-42, 1968.

[11] T. J. R. Hughes, J. A. Cottrell and Y. Bazilevs "Isogeometric analysis: CAD, finite elements, NURBS, exact geometry and mesh refinement". Computer Methods in Applied Mechanics and Engineering, vol. 194, Issues. 39-41, p. 4135-4195, 2005.

[12] X. Gu, Y. He, H. Qin, Manifold splines. Graphical Models, vol. 68, p. 237-254, 2006.

[13] Y. He, K. Wang, H. Wang, X. Gu, H. Qin, Manifold T-spline. Geometric Modeling and Processing-GMP 2006, Lecture Notes in Computer Science, vol. 4077, p. 409-422, 2006.

[14] H. Wang, Y. He, X. Li, X. Gu, H. Qin, Polycube Splines. Computer-Aided Design, vol. 40, p. 721-733, 2008.

[15] L. Ying, D. Zorin, A simple manifold-based construction of surfaces of arbitrary smoothness. ACM Trans. Graph., vol. 23, p. 271-275, 2004.

[16] J. Peter, U. Reif, Subdivision Surfaces. Geometry and Computing, Springer-Verlag, ISBN 878-3540-76406-9, 2008.

[17] J. A. Gregory Smooth interpolation without twist constraints. Waltham,Massachusetts: AcademicPress, p. 71-88, 1974.

[18] T. DeRose, C. Loop. The S-patch: a new multisided patch scheme. ACM TransGraph, vol. 8, p. 204-233, 1989.

[19] B. R. Piper. Visually smooth interpolation with triangular Bézier patches. In: G. Farin, editor. Geometric modeling. Philadelphia: SIAM, p. 221-233, 1987. 
[20] J. Peter. Smooth free-form surfaces over irregular meshes generalizing quadratic splines. Computer Aided Geometric Design, vol. 10, p. 347-361, 1993.

[21] J. Peter. $C^{1}$-surface splines. SIAM J. Numer Anal, vol. 32, p. 645-666, 1995.

[22] E. Catmull, J. Clark. Recursively generated B-spline surfaces on arbitrary topological meshes. Computer Aided Geometric Design, vol. 10, p. 350-355, 1978.

[23] U. Reif. Biquadratic G-spline surfaces. Computer Aided Geometric Design, vol. 12, p. 193-205, 1995.

[24] U. Reif. A refineable space of smooth spline surfaces of arbitrary topological genus. Journal of Approximation Theory, vol. 90, p. 174-199, 1997.

[25] J. Deng, F. Chen, and Y. Feng. Dimensions of spline spaces over T-meshes. Journal of Computational and Applied Mathematics, vol. 194, p. 267-283, 2006.

[26] L. L. Schumaker and L. Wang. Approximation power of polynomial splines on T-meshes Computer Aided Geometric Design 29, p. 599-612, 2012

[27] T. Takacs, B. Jüttler. "Existence of Stiffness Matrix Integrals for Singularly Parameterized Domains in Isogeometric Analysis". Computer Methods in Applied Mechanics and Engineering, vol. 200, p. 3568-3582, 2011.

[28] A. Quarteroni, A. Valli. Numerical Approximation of Partial Differential Equations. Springer-Verlag, ISBN 3-540-57111-6, 1997.

[29] L. L. Schumaker, L. Wang. On Hermite interpolation with polynomial splines on T-meshes. Journal of Computational and Applied Mathematics, vol. 240, p. 42-50, 2013.

[30] A. Pataki, A. J. Cerfon, J. P. Freidberg, L. Greengard and M. O. Neil. A fast, high-order solver for the Grad-Shafranov equation. J. Comput. Phys., vol. 243, p. 28-45, 2013.

[31] B. Mourrain. On the dimension of spline spaces on planar T-meshes. Mathematics of Computation, 83, p. 847-871, 2014.

[32] R. L. Burden, J. D. Faires. Numerical Analysis. Prindle, Weber and Schnidt, Boston, MA 1985.

[33] B. Mourrain, R. Vidunas and N. Villamizar Dimension and bases for geometrically continuous splines on surfaces of arbitrary topology. Computer Aided Geometric Design, in press, 2016. 